\title{
High-accuracy comparison of numerical relativity simulations with post-Newtonian expansions
}

\author{
Michael Boyle, ${ }^{1}$ Duncan A. Brown, ${ }^{1,2,3}$ Lawrence E. Kidder, ${ }^{4}$ Abdul H. Mroué, ${ }^{4}$ Harald P. Pfeiffer, ${ }^{1}$ Mark A. Scheel, ${ }^{1}$ \\ Gregory B. Cook, ${ }^{5}$ and Saul A. Teukolsky ${ }^{4}$ \\ ${ }^{1}$ Theoretical Astrophysics 130-33, California Institute of Technology, Pasadena, California 91125, USA \\ ${ }^{2}$ LIGO Laboratory, California Institute of Technology, Pasadena, California 91125, USA \\ ${ }^{3}$ Department of Physics, Syracuse University, Syracuse, New York, 13244, USA \\ ${ }^{4}$ Center for Radiophysics and Space Research, Cornell University, Ithaca, New York, 14853, USA \\ ${ }^{5}$ Department of Physics, Wake Forest University, Winston-Salem, North Carolina 27106, USA
}

(Received 30 September 2007; published 27 December 2007)

\begin{abstract}
Numerical simulations of 15 orbits of an equal-mass binary black-hole system are presented. Gravitational waveforms from these simulations, covering more than 30 cycles and ending about 1.5 cycles before merger, are compared with those from quasicircular zero-spin post-Newtonian (PN) formulae. The cumulative phase uncertainty of these comparisons is about 0.05 radians, dominated by effects arising from the small residual spins of the black holes and the small residual orbital eccentricity in the simulations. Matching numerical results to PN waveforms early in the run yields excellent agreement (within 0.05 radians) over the first $\sim 15$ cycles, thus validating the numerical simulation and establishing a regime where PN theory is accurate. In the last 15 cycles to merger, however, generic time-domain Taylor approximants build up phase differences of several radians. But, apparently by coincidence, one specific post-Newtonian approximant, TaylorT4 at 3.5PN order, agrees much better with the numerical simulations, with accumulated phase differences of less than 0.05 radians over the 30-cycle waveform. Gravitational-wave amplitude comparisons are also done between numerical simulations and postNewtonian, and the agreement depends on the post-Newtonian order of the amplitude expansion: the amplitude difference is about $6 \%-7 \%$ for zeroth order and becomes smaller for increasing order. A newly derived 3.0PN amplitude correction improves agreement significantly $(<1 \%$ amplitude difference throughout most of the run, increasing to $4 \%$ near merger) over the previously known $2.5 \mathrm{PN}$ amplitude terms.
\end{abstract}

DOI: 10.1103/PhysRevD.76.124038

PACS numbers: 04.25.Nx, 04.25.D-, 04.25.dg, 04.30.-w

\section{INTRODUCTION}

The last two years have witnessed tremendous progress in simulations of black-hole binaries, starting with the first stable simulation of orbiting and merging black holes [1,2], development of the moving puncture method $[3,4]$, and rapid progress by other groups [5-13]. Since then, an enormous amount of work has been done on the late inspiral and merger of black-hole binaries, among them studies of the universality of the merger waveforms [14,15], investigations into black-hole kicks [11,16-28], and spin dynamics [29-31], comparisons to postNewtonian (PN) models [32-34], and applications to gravitational-wave data analysis [35-37].

Compared to the intense activity focusing on simulations close to merger, there have been relatively few simulations covering the inspiral phase. To date, only three simulations [38-42] cover more than five orbits. Long inspiral simulations are challenging for a variety of reasons: First, the orbital period increases rapidly with separation, so that simulations must cover a significantly longer evolution time. In addition, the gravitational waveform must be extracted at larger radius (and the simulation must therefore cover a larger spatial volume) because the gravitational wavelength is longer. Furthermore, gravitational-wave data analysis requires small absolute accumulated phase uncertainties in the waveform, so the relative phase uncertainty of the simulation must be smaller.

Gravitational-wave detectors provide a major driving force for numerical relativity (NR). The first generation interferometric gravitational-wave detectors, such as LIGO [43,44], GEO600 [45], and VIRGO [46,47], are now operating at or near their design sensitivities. Furthermore, the advanced generation of detectors are entering their construction phases. This new generation of interferometers will improve detector sensitivity by a factor of $\sim 10$ and hence increase expected event rates by a factor of $\sim 1000$ [48]. One of the most promising sources for these detectors is the inspiral and merger of binary black holes (BBHs) with masses $m_{1} \sim m_{2} \sim 10-20 M_{\odot}$ [49]. These systems are expected to have circularized long before their gravitational waves enter the sensitive frequency band of ground-based detectors [50].

A detailed and accurate understanding of the gravitational waves radiated as the black holes spiral towards each other will be crucial not only to the initial detection of such sources, but also to maximize the information that can be obtained from signals once they are observed. When the black holes are far apart, the gravitational wave- 
form can be accurately computed using a PN expansion. As the holes approach each other and their velocities increase, the post-Newtonian expansion is expected to diverge from the true waveform. It is important to quantify any differences between theoretical waveforms and the true signals, as discrepancies will cause a reduction of search sensitivity. Several techniques have been proposed to address the problem of the breakdown of the postNewtonian approximation [51-53], but ultimately, the accuracy of the post-Newtonian waveforms used in binary black-hole gravitational-wave searches can only be established through comparisons with full numerical simulations.

Unfortunately, comparing post-Newtonian approximations to numerical simulations is not straightforward, the most obvious problem being the difficulty of producing long and sufficiently accurate numerical simulations as explained above. In addition, post-Newtonian waveforms typically assume circular orbits, and most astrophysical binaries are expected to be on circular orbits late in their inspiral, so the orbital eccentricity within the numerical simulation must be sufficiently small. ${ }^{1}$ Another factor that complicates comparisons is the variety of post-Newtonian approximants available, from several straightforward Taylor expansions to more sophisticated Padé resummation techniques and the effective one-body approach (see e.g. [51,52,54-60], as well as Sec. III E below). While all post-Newtonian approximants of the same order should agree sufficiently early in the inspiral (when neglected higher-order terms are small), they begin to disagree with each other during the late inspiral when the postNewtonian approximation starts to break down-exactly the regime in which NR waveforms are becoming available.

Finally, agreement (or disagreement) between NR and PN waveforms will also depend very sensitively on the precise protocol used to compare the waveforms. Are PN and NR waveforms matched early or late in the inspiral? Is the matching done at a particular time, or is a least-squares fit performed over part (or all) of the waveform? Does one compare frequencies $\omega(t)$ or phases $\phi(t)$ ? Are comparisons presented as functions of time or of frequency? Up to which cutoff frequency does one compare PN with NR?

Despite these difficulties, several comparisons between NR and PN have been done for the last few orbits of an equal-mass, nonspinning black-hole binary. The first such study was done by Buonanno et al. [32] based on simulations performed by Pretorius [1] lasting somewhat more than 4 orbits ( $\sim 8$ gravitational-wave cycles). This comparison performs a least-squares fit over the full waveform, finds agreement between the numerical evolution and a

\footnotetext{
${ }^{1}$ Unfortunately, this circularization occurs on extremely long time scales [50], thousands of orbits, making it impossible to run the numerical simulation long enough to radiate the eccentricity away.
}

particular post-Newtonian approximant (in our language TaylorT3 3.0/0.0 ${ }^{2}$ ) and notes that another approximant (TaylorT4 3.5/0.0) will give similarly good agreement. However, as the authors note, this study is severely limited by numerical resolution, sizable initial eccentricity $(\sim$ 0.015), close initial separation of the black holes, and coordinate artifacts; for these reasons, the authors do not quantify the level of agreement.

More recently, Baker et al. [38,39] performed simulations covering the last $\sim 14$ cycles before merger. These simulations have an orbital eccentricity $\sim 0.008$ [38], forcing the authors to use a fitted smooth ("deeccentrized") gravitational-wave phase to obtain a monotonically increasing gravitational-wave frequency. Comparing to TaylorT4 3.5/2.5, they find agreement between the numerical and post-Newtonian gravitational-wave phase to within their numerical errors, which are about 2 radians. The authors also indicate that other post-Newtonian approximants do not match their simulation as well as TaylorT4, but unfortunately, they do not mention whether any disagreement is significant (i.e., exceeding their numerical errors). Pan et al. [35] performed a more comprehensive analysis of the numerical waveforms computed by Pretorius [32] and the Goddard group [38,39], confirming that TaylorT4 $3.5 / 0.0$ matches the numerical results best.

The most accurate inspiral simulation to date was performed by the Jena group and presented in Husa et al. [42] and Hannam et al. [41]. This simulation covers 18 cycles before merger and has an orbital eccentricity of $\sim 0.0018$ [61]. Discarding the first two cycles which are contaminated by numerical noise, and terminating the comparison at a gravitational-wave frequency $m \omega=0.1$ (see Eq. (15) for the precise definition) their comparison extends over 13 cycles. We discuss the results of Ref. [41] in more detail in Sec. VI A 1.

This paper presents a new inspiral simulation of a nonspinning equal-mass black-hole binary. This new simulation more than doubles the evolution time of the simulations in Refs. [38,39,41,42], resulting in a waveform with 30 gravitational-wave cycles, ending $\sim 1.5$ cycles before merger, and improves numerical truncation errors by 1 to 2 orders of magnitude over those in Refs. [38,39,41,42]. The orbital eccentricity of our simulations is $\sim 6 \times 10^{-5}$; this low eccentricity is achieved using refinements of techniques described in [40]. We present a detailed analysis of various effects which might influence our comparisons to post-Newtonian waveforms for nonspinning black-hole binaries on circular orbits. These effects result in an uncertainty of $\sim 0.05$ radians out of the accumulated $\sim 200$ radians. Perhaps surprisingly,

\footnotetext{
${ }^{2}$ We identify post-Newtonian approximants with three pieces of information: the label introduced by [54] for how the orbital phase is evolved; the PN order to which the orbital phase is computed; and the PN order that the amplitude of the waveform is computed. See Sec. III E for more details.
} 
the largest uncertainty arises from the residual orbital eccentricity, despite its tiny value. The second largest effect arises due to a potential residual spin on the black holes, which we bound by $|S| / M_{\text {irr }}^{2}<5 \times 10^{-4}$.

We compare the numerical waveforms with four different time-domain post-Newtonian Taylor-approximants [53-55] and we match PN and NR waveforms at a specific time during the inspiral. We explore the effects of varying this matching time. When matching $\sim 9$ cycles after the start of our evolution, all post-Newtonian approximants of 3.0PN and 3.5PN order in orbital phase agree with our simulation to within $\sim 0.03$ radians over the first 15 cycles. This agreement is better than the combined uncertainties of the comparison, thus validating our simulations in a regime where the 3.5PN truncation error of post-Newtonian theory is comparable to the accuracy of our simulations. Lower order post-Newtonian approximants (2.0PN and 2.5PN order), however, accumulate a significant phase difference of $\sim 0.2$ radians over this region.

Extending the comparison toward merger (as well as when matching closer to merger), we find, not surprisingly, that the agreement between PN and NR at late times depends strongly on exactly what post-Newtonian approximant we use [54,55]. Typical accumulated phase differences are on the order of radians at frequency $m \omega=0.1$. One particular post-Newtonian approximant, TaylorT4 at 3.5PN order in phase, agrees with our NR waveforms far better than the other approximants, the agreement being within the phase uncertainty of the comparison ( 0.05 radians) until after the gravitational-wave frequency passes $m \omega=0.1$ (about 3.5 cycles before merger). It remains to be seen whether this agreement is fundamental or accidental, and whether it applies to more complicated situations (e.g. unequal masses, nontrivial spins).

We also compare the post-Newtonian gravitationalwave amplitude to the numerical amplitude, where we estimate the uncertainty of this comparison to be about $0.5 \%$. Restricted waveforms (i.e., 0PN order in the amplitude expansion) are found to disagree with the numerical amplitudes by $6 \%-7 \%$. An amplitude expansion of order 2PN shows significantly better agreement than the expansion at order 2.5PN. A newly derived 3PN amplitude [62] is found to give much better agreement than the 2.0PN amplitude.

This paper is organized as follows: Sec. II discusses our numerical techniques. In particular, we describe how we construct binary black-hole initial data, evolve these data for 15 orbits, extract gravitational-wave information from the evolution, and produce a gravitational waveform as seen by an observer at infinity. Section III details the generation of post-Newtonian waveforms, including details of how we produce the four approximants that we compare against NR. We describe our procedure for comparing NR and PN waveforms in Sec. IV, and present a detailed study of various sources of uncertainty in Sec. V.
The comparisons between NR and PN are presented in Sec. VI. This section is split into two parts: First, we compare each PN approximant separately with the numerical simulation. Subsequently, we show some additional figures which facilitate cross-comparisons between the different PN approximants. Finally, we present some concluding remarks in Sec. VII. The impatient reader primarily interested in NR-PN comparisons may wish to proceed directly to Table III summarizing the uncertainties of our comparisons, and then continue to Sec. VI, starting with Fig. 15.

\section{GENERATION OF NUMERICAL WAVEFORMS}

In order to do a quantitative comparison between numerical and post-Newtonian waveforms, it is important to have a code capable of starting the black holes far enough apart to be in a regime where we strongly believe the postNewtonian approximation is valid, track the orbital phase extremely accurately, and do so efficiently so the simulation can be completed in a reasonable amount of time. Furthermore, the gravitational waves from such a simulation must be extracted in such a manner that preserves the accuracy of the simulation and predicts the waveform as seen by a distant observer, so a comparison with the postNewtonian waveform can be made. In this section we describe the techniques we use to do this, as well as the results of a simulation starting more than 15 orbits prior to merger.

When discussing numerical solutions of Einstein's equations, we write all dimensioned quantities in terms of some mass scale $m$, which we choose to be the sum of the irreducible masses of the two black holes in the initial data:

$$
m=M_{\mathrm{irr}, 1}+M_{\mathrm{irr}, 2} \text {. }
$$

The irreducible mass of a single hole is defined as

$$
M_{\text {irr }} \equiv \sqrt{A / 16} \pi,
$$

where $A$ is the surface area of the event horizon; in practice we take $A$ to be the surface area of the apparent horizon. More generally, it is more appropriate to use the Christodoulou mass of each black hole,

$$
M_{\mathrm{BH}}^{2}=M_{\mathrm{irr}}^{2}+\frac{S^{2}}{4 M_{\mathrm{irr}}^{2}},
$$

instead of the irreducible mass. Here $S$ is the spin of the hole. However, for the case considered in this paper, the spins are sufficiently small and there is little difference between $M_{\mathrm{BH}}$ and $M_{\text {irr }}$.

\section{A. Initial data}

Initial data are constructed within the conformal thin sandwich formalism $[63,64]$ using a pseudospectral elliptic solver [65]. We employ quasiequilibrium boundary conditions $[66,67]$ on spherical excision boundaries, choose 
conformal flatness and maximal slicing, and use Eq. (33a) of Ref. [68] as the lapse boundary condition. The spins of the black holes are made very small via an appropriate choice of the tangential shift at the excision surfaces, as described in [68].

As the most accurate post-Newtonian waveforms available assume adiabatic inspiral of quasicircular orbits, it is desirable to reduce the eccentricity of the numerical data as much as possible. Using techniques developed in [40], each black hole is allowed to have a nonzero initial velocity component towards the other hole. This small velocity component $v_{r}$ and the initial orbital angular velocity $\Omega_{0}$ are then fine-tuned in order to produce an orbit with very small orbital eccentricity. ${ }^{3}$ We have improved our eccentricity-reduction procedure since the version described in [40], so we summarize our new iterative procedure here:

We start with a quasicircular (i.e., $v_{r}=0$ ) initial data set at coordinate separation $d=30$, where $\Omega_{0}$ is determined by equating Komar mass with Arnowitt-Deser-Misner (ADM) mass [68]. We then evolve these data for about 1.5 orbits, corresponding to a time $t / m \approx 600$. From this short evolution, we measure the proper separation $s$ between the horizons by integration along the coordinate axis connecting the centers of the black holes. We fit the time derivative $d s / d t$ in the interval $100 \leqq t / m \lesssim 600$ to the function

$$
\frac{d s}{d t}=A_{0}+A_{1} t+B \cos (\omega t+\varphi),
$$

where we vary all five parameters $A_{0}, A_{1}, B, \omega$, and $\varphi$ to achieve the best fit. The desired smooth inspiral is represented by the part $A_{0}+A_{1} t$; the term $B \cos (\omega t+\varphi)$ corresponds to oscillations caused by orbital eccentricity.

For a Newtonian orbit with radial velocity $B \cos (\omega t+$ $\varphi$ ) at initial separation $s_{0}$, it is straightforward to determine the changes to the orbital frequency and the radial velocity which make the orbit perfectly circular, namely

$$
\begin{gathered}
\Omega_{0} \rightarrow \Omega_{0}+\frac{B \sin \varphi}{2 s_{0}}, \\
v_{r} \rightarrow v_{r}-\frac{B \cos \varphi}{2} .
\end{gathered}
$$

For Newtonian gravity, Eq. (6) will of course result in a circular orbit with $v_{r}=0$. In general relativity, $\Omega_{0}$ and $v_{r}$ will be different from their Newtonian values, for instance $v_{r}<0$ to account for the inspiral of the two black holes. Nevertheless, we assume that small perturbations around

\footnotetext{
${ }^{3}$ An alternative method of producing low-eccentricity initial data, based on post-Newtonian ideas, is developed in [61]. While that technique is computationally more efficient than ours, it merely reduces orbital eccentricity by a factor of $\sim 5$ relative to quasicircular initial data, which is insufficient for the comparisons presented here (cf. Sec. VE 2).
}

the zero-eccentricity inspiral trajectory behave similarly to small perturbations around a Newtonian circular orbit. Therefore, we apply the same formulae, Eqs. (5) and (6), to obtain improved values for $\Omega_{0}$ and $v_{r}$ for the black-hole binary, where $s_{0}$ is the initial proper separation between the horizons. We then use the new values of $\Omega_{0}$ and $v_{r}$ to construct a new initial data set, again evolve for two orbits, fit to Eq. (4), and update $\Omega_{0}$ and $v_{r}$. We continue iterating this procedure until the eccentricity is sufficiently small.

We estimate the eccentricity for each iteration from the fit to Eq. (4) using the formula

$$
e_{d s / d t}=\frac{B}{s_{0} \omega},
$$

which is valid in Newtonian gravity for small eccentricities. Successive iterations of this procedure are illustrated in Fig. 1 and yield the initial data sets 30a, 30b, and 30c summarized in Table I. Eccentricity decreases by roughly a factor of 10 in each iteration, with $30 \mathrm{c}$ having $e_{d s / d t} \approx$ $5 \times 10^{-5}$. The evolutions used during eccentricity reduction need not be very accurate and need to run only for a short time, $t \sim 600 \mathrm{~m}$. One iteration of this procedure at our second lowest resolution requires about 250 CPU-hours. For completeness, Table I also lists parameters for initial data at smaller separation; these data will be used for consistency checks below. Apart from these consistency checks, the remainder of this paper will focus exclusively on evolutions of the low-eccentricity initial data set 30c.

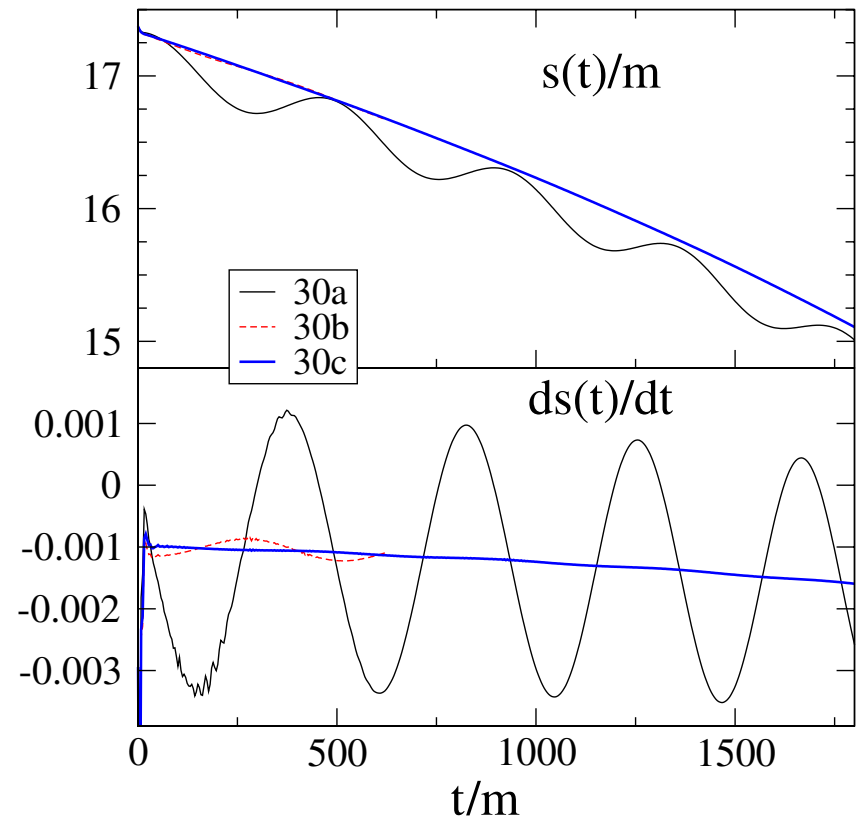

FIG. 1 (color online). Proper separation (top panel) and its time derivative (lower panel) versus time for short evolutions of the $d=30$ initial data sets 30a, 30b, and 30c (see Table I). These three data sets represent zero through two iterations of our eccentricity-reduction procedure. The orbital eccentricity is reduced significantly by each iteration. 
TABLE I. Summary of the initial data sets used in this paper. The first block of numbers $\left(d, \Omega_{0}, f_{r}\right.$, and $\left.v_{r}\right)$ represent raw parameters entering the construction of the initial data. The second block gives some properties of each initial data set: $m$ denotes the sum of the irreducible masses, $M_{\mathrm{ADM}}$ and $J_{\mathrm{ADM}}$ the $\mathrm{ADM}$ energy and angular momentum, and $s_{0}$ the initial proper separation between the horizons. The last column lists the eccentricity computed from Eq. (7). The initial data set 30c is used for all evolutions (except for consistency checks) described in this paper.

\begin{tabular}{lccccccccr}
\hline \hline Name & $d$ & $\Omega_{0}$ & $f_{r}$ & $v_{r} \times 10^{4}$ & $m \Omega_{0}$ & $M_{\mathrm{ADM}} / m$ & $J_{\mathrm{ADM}} / m^{2}$ & $s_{0} / m$ \\
\hline 30a & 30 & 0.0080108 & 0.939561 & 0.00 & 0.01664793 & 0.992333 & 1.0857 & 17.37 & $1.0 \times 10^{-2}$ \\
30b & 30 & 0.0080389 & 0.939561 & -4.90 & 0.0167054 & 0.992400 & 1.0897 & 17.37 & $6.5 \times 10^{-4}$ \\
30c & 30 & 0.0080401 & 0.939561 & -4.26 & 0.0167081 & 0.992402 & 1.0898 & 17.37 & $5 \times 10^{-5}$ \\
$24 \mathrm{a}$ & 24 & 0.0110496 & 0.92373 & -8.29 & 0.0231947 & 0.990759 & 1.0045 & 14.15 & $1.1 \times 10^{-3}$ \\
24b & 24 & 0.0110506 & 0.923739 & -8.44 & 0.0231967 & 0.990767 & 1.0049 & 14.15 & $1.5 \times 10^{-4}$ \\
\hline \hline
\end{tabular}

\section{B. Evolution of the inspiral phase}

The Einstein evolution equations are solved with the pseudospectral evolution code described in Ref. [7]. This code evolves a first-order representation [69] of the generalized harmonic system [70-72]. We handle the singularities by excising the black-hole interiors from our grid. Our outer boundary conditions $[69,73,74]$ are designed to prevent the influx of unphysical constraint violations [75-81] and undesired incoming gravitational radiation [82], while allowing the outgoing gravitational radiation to pass freely through the boundary.

The code uses a fairly complicated domain decomposition to achieve maximum efficiency. Each black hole is surrounded by several (typically six) concentric spherical shells, with the inner boundary of the innermost shell (the excision boundary) just inside the horizon. A structure of touching cylinders (typically 34 of them) surrounds these shells, with axes along the line between the two black holes. The outermost shell around each black hole overlaps the cylinders. The outermost cylinders overlap a set of outer spherical shells, centered at the origin, which extend to large outer radius. External boundary conditions are imposed only on the outer surface of the largest outer spherical shell. We vary the location of the outer boundary by adding more shells at the outer edge. Since all outer shells have the same angular resolution, the cost of placing the outer boundary farther away (at full resolution) increases only linearly with the radius of the boundary. External boundary conditions are enforced using the method of Bjørhus [83], while interdomain boundary conditions are enforced with a penalty method [84,85].

We employ the dual-frame method described in Ref. [7]: we solve the equations in an "inertial frame" that is asymptotically Minkowski, but our domain decomposition is fixed in a "comoving frame" that rotates with respect to the inertial frame and also shrinks with respect to the inertial frame as the holes approach each other. The positions of the holes are fixed in the comoving frame; we account for the motion of the holes by dynamically adjusting the coordinate mapping between the two frames. Note that the comoving frame is referenced only internally in the code as a means of treating moving holes with a fixed domain. Therefore all coordinate quantities (e.g. blackhole trajectories, wave-extraction radii) mentioned in this paper are inertial-frame values unless explicitly stated otherwise.

One side effect of our dual-frame system is that the outer boundary of our domain (which is fixed in the comoving frame) moves inward with time as observed in the inertial frame. This is because the comoving frame shrinks with respect to the inertial frame to follow the motion of the holes. In Refs. [7,40] the inertial-frame coordinate radius $r$ (with respect to the center of mass) and the comoving coordinate radius $r^{\prime}$ are related by a simple scaling

$$
r=a(t) r^{\prime}
$$

The expansion parameter $a(t)$ is initially set to unity and decreases dynamically as the holes approach each other, so that the comoving-frame coordinate distance between the holes remains constant. The outer boundary of the computational grid is at a fixed comoving radius $R_{\text {bdry }}^{\prime}$, which is mapped to the inertial coordinate radius $R_{\text {bdry }}(t)=$ $a(t) R_{\text {bdry }}^{\prime}$. Because we wish to accurately compute the gravitational radiation as measured far from the holes, it is desirable to have a moderately large outer boundary ( $\left.R_{\text {bdry }}(t) \gtrsim 200 m\right)$ throughout the run. For the linear mapping, Eq. (8), this requires a very distant outer boundary early in the run, $R_{\text {bdry }}(0) \simeq 1000 m$. Computationally this is not very expensive. However, the initial junk radiation contaminates the evolutions for a time interval proportional to the light-crossing time to the outer boundary, and for $R_{\text {bdry }}(0) \simeq 1000 m$ it would be necessary to discard a significant portion of the evolution.

We therefore use the mapping

$$
r=\left[a(t)+(1-a(t)) \frac{r^{\prime 2}}{R_{0}^{\prime 2}}\right] r^{\prime},
$$

for some constant $R_{0}^{\prime}$ which is chosen to be roughly the radius of the outer boundary in comoving coordinates. This mapping has the following properties: (1) At the initial time $t=0$, the map reduces to the identity map because $a(0)=1$. Thus we do not need to remap our initial data before evolving. (2) For small radii (i.e., at the locations of 
TABLE II. Overview of low-eccentricity simulations discussed in this paper. $R_{\text {bdry }}$ is the initial coordinate radius of the outer boundary; this radius changes during the evolution according to the choice of "radial map" between inertial and comoving coordinates. The last column lists the different resolutions run for each evolution, N6 being highest resolution. Evolution 30c-1/ N6 forms the basis of our post-Newtonian comparisons, and is used in all figures unless noted otherwise.

\begin{tabular}{cccccc}
\hline \hline Name & ID & $N_{\text {orbits }}$ & $R_{\text {bdry }}$ & Radial map & Resolutions \\
\hline 30c-1 & 30c & 15.6 & $462 m$ & Equation (9) & $\mathrm{N} 1, \mathrm{~N} 2, \ldots, \mathrm{N} 6$ \\
30c-2 & 30c & 15.6 & $722 m$ & Equation (8) & $\mathrm{N} 2, \mathrm{~N} 4, \mathrm{~N} 6$ \\
30c-3 & 30c & 15.6 & $202 m$ & Equation (8) & $\mathrm{N} 2, \mathrm{~N} 3, \ldots, \mathrm{N} 6$ \\
$24 \mathrm{~b}-1$ & $24 \mathrm{~b}$ & 8.3 & $160 m$ & Equation (8) & $\mathrm{N} 2, \mathrm{~N} 3, \mathrm{~N} 4$ \\
\hline \hline
\end{tabular}

the black holes), the map reduces to the linear map, $r=$ $a(t) r^{\prime}+\mathcal{O}\left(r^{\prime 3}\right)$. This allows use of the control system without modifications. (3) The moving radius $r^{\prime}=R_{0}^{\prime}$ is mapped to a constant inertial radius: $r\left(R_{0}^{\prime}\right)=R_{0}^{\prime}$. This allows us to keep the inertial radius of the outer boundary constant (or nearly constant ${ }^{4}$ ) in time rather than shrinking rapidly.

In total, we have run three evolutions of the $30 \mathrm{c}$ initial data set; these use different combinations of outer boundary radius and radial mapping between inertial and moving coordinates. Some properties of these evolutions are summarized in Table II. We also performed extensive convergence testing, running the same evolution on up to six distinct resolutions, N1 to N6. The coarsest resolution $30 \mathrm{c}-1 / \mathrm{N} 1$ uses approximately $41^{3}$ grid points (summing all grid points in all the subdomains), while the most accurate evolution, 30c-1/N6, uses about $67^{3}$ grid points. The run $30 \mathrm{c}-1 / \mathrm{N} 2$ required about $2500 \mathrm{CPU}$-hours and run $30 \mathrm{c}-1 / \mathrm{N} 6$ about 19000 , where our simulations do not take advantage of symmetries. The distance to the outer boundary is adjusted by adding or removing outer spherical shells to an otherwise unmodified domain decomposition. Run $30 \mathrm{c}-1$ has 20 such outer spherical shells, while 30c-2 utilizes 32 and $30 \mathrm{c}-3$ only 8 . Thus, the total number of grid points varies slightly between runs, e.g. about $71^{3}$ for $30 \mathrm{c}-2 / \mathrm{N} 6$. Figure 2 indicates the different behavior of the outer boundary location for these three evolutions.

For all of the evolutions $30 \mathrm{c}-1 / 2 / 3$, the coordinate trajectories of the centers of the apparent horizons appear as in Fig. 3. The regular inspiral pattern without noticeable oscillations once again indicates that our evolutions indeed have very low eccentricity.

Figure 4 demonstrates the convergence of the black-hole mass $m(t)$ with spatial resolution for run $30 \mathrm{c}-1$. The mass $m(t)$ is computed as the sum of the irreducible masses of

\footnotetext{
${ }^{4}$ In practice, we choose $R_{0}^{\prime}$ somewhat larger than the outer boundary, so that the outer boundary of the computational domain slowly contracts in inertial coordinates. This makes the zero-speed characteristic fields outgoing there, avoiding the need to impose boundary conditions on those fields.
}

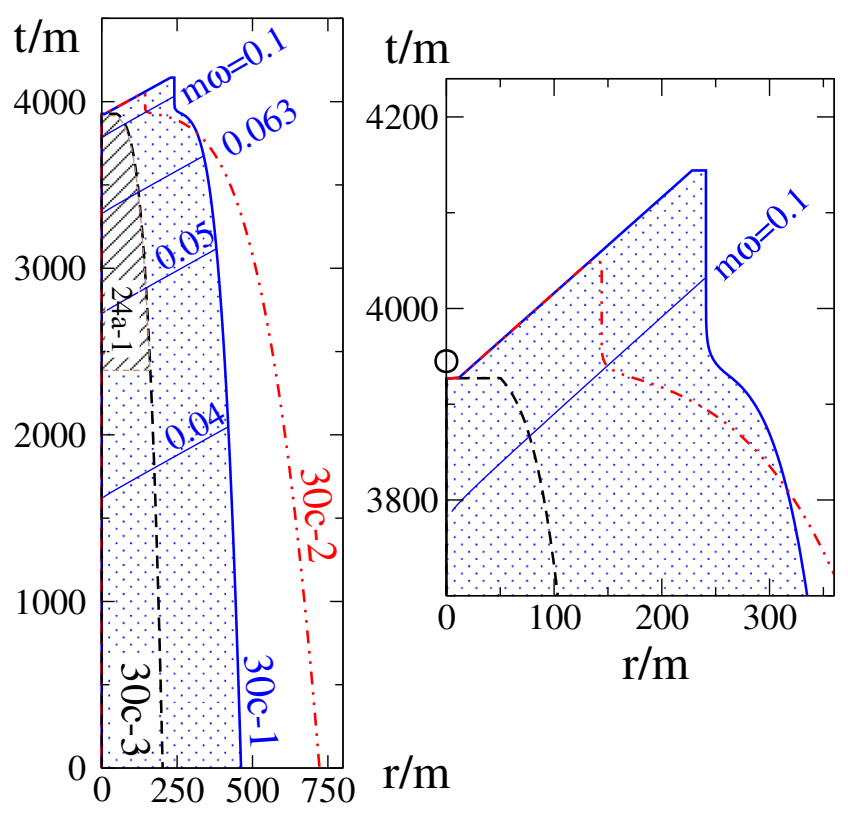

FIG. 2 (color online). Spacetime diagram showing the spacetime volume simulated by the numerical evolutions listed in Table II. The magnified view in the right panel shows how the gravitational waves are escorted to our extraction radii (see Sec. II C) after the simulation in the center has already crashed at $t \sim 3930 \mathrm{~m}$, and after the estimated time of the black-hole merger, which is indicated by the circle. The thin diagonal lines are lines of constant $t-r^{*}$; each corresponds to a retarded time at which the gravitational-wave frequency $\omega$ at infinity assumes a particular value.

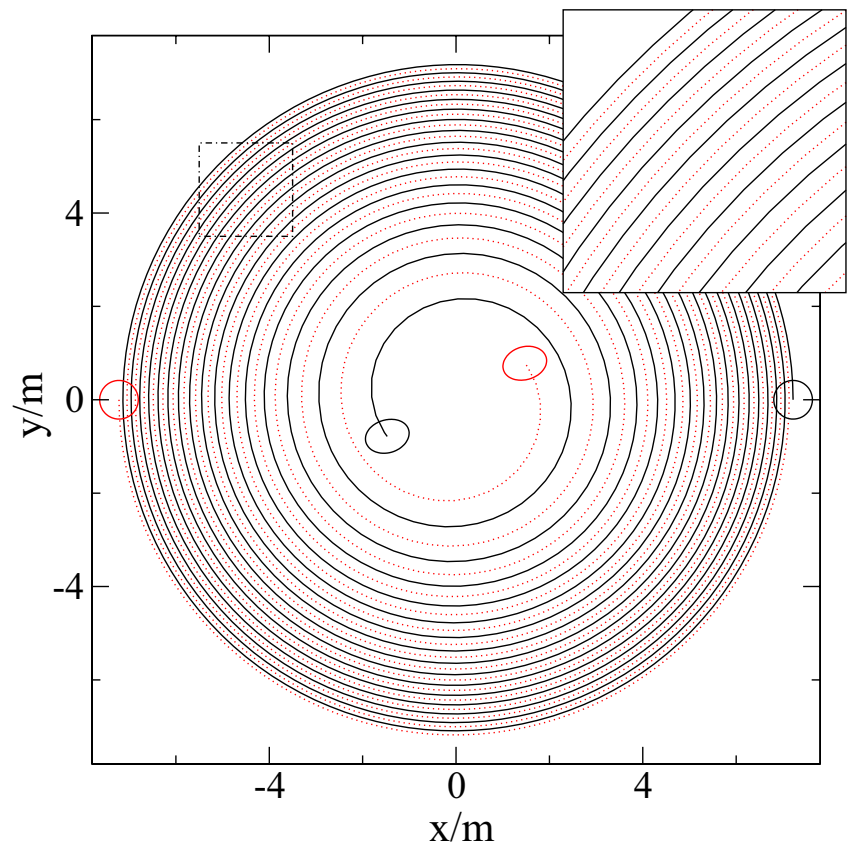

FIG. 3 (color online). Coordinate trajectories of the centers of the black holes. The small circles/ellipsoids show the apparent horizons at the initial time and at the time when the simulation ends and wave escorting begins. The inset shows an enlargement of the dashed box. 


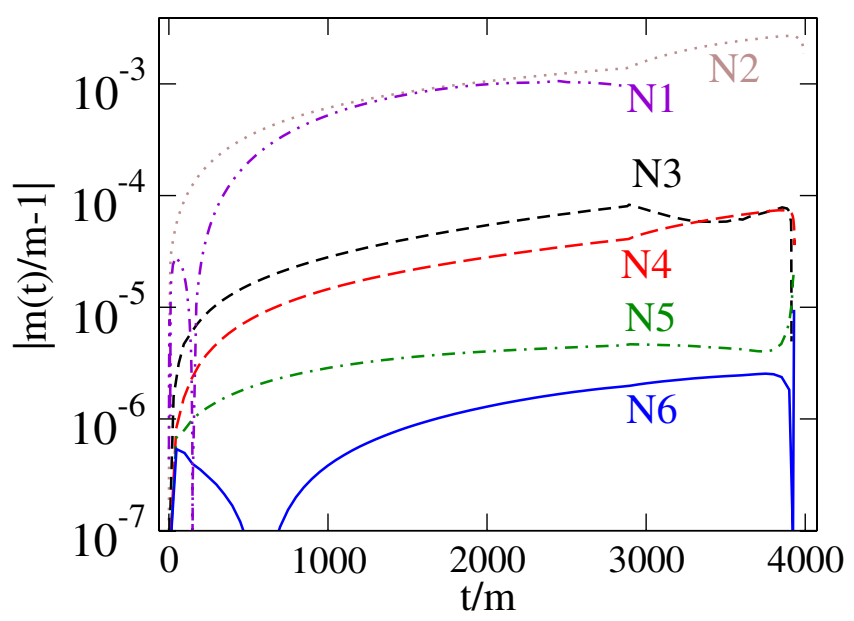

FIG. 4 (color online). Deviation of total irreducible mass $m(t)=2 M_{\text {irr }}(t)$ from its value in the initial data. Plotted are the six different resolutions of run $30 \mathrm{a}-1$.

both black holes, as defined in Eq. (2). At the highest resolution, $m(t)$ deviates by only a few parts in $10^{6}$ from its initial value $m$.

Our apparent horizon finder works by expanding the radius of the apparent horizon as a series in spherical harmonics up to some order $L$. We utilize the fast flow methods developed by Gundlach [86] to determine the expansion coefficients; these are significantly faster than our earlier minimization algorithms $[87,88]$. The apparent horizon is almost spherical during the inspiral, so that the expansion in $L$ converges exceedingly fast: $L=8$ results in a relative error of the irreducible mass of better than $10^{-8}$. The distortion of the horizons becomes more pronounced toward the end of the evolution when the black holes approach each other rapidly. This results in an error of $10^{-6}$ in the $L=8$ apparent horizon expansion for the last $10 \mathrm{~m}$ of the evolution.

We also measure the quasilocal spin using coordinate rotation vectors projected into the apparent horizon surfaces [89-91]. Only the $z$-component of the spin is nonzero (i.e., the spins are aligned with the orbital angular momentum). The spin starts at $S_{z} / M_{\text {irr }}^{2} \approx-6 \times 10^{-5}$ and increases slowly to $-5 \times 10^{-4}$ during the evolution, where the minus sign indicates that the black-hole spin is antialigned with the orbital angular momentum. Thus it appears the black hole's spins move further away from the corotational state. We believe this effect is caused by the use of coordinate rotation vectors when calculating the quasilocal spin, rather than more sophisticated approximate Killing vectors [92-94]. Preliminary results with approximate Killing vectors find the initial spin to be less than $10^{-6}$, and slowly increasing during the evolution to a final value of $2 \times 10^{-5}$ at the end of the comparison interval to post-Newtonian theory. Given the preliminary character of these results, we will take here the conservative bound $|\mathbf{S}| / M_{\mathrm{irr}}^{2} \leq 5 \times 10^{-4}$ obtained from coordinate rotation vectors.

\section{Escorting gravitational waves}

The simulation presented in Fig. 3 stops when the horizons of the black holes become too distorted just before merger. At this time, most of the domain (all regions except for the immediate vicinity of the two holes) is still well resolved, and the spacetime contains gravitational radiation that has not yet propagated out to the large radii where we perform wave extraction. So instead of losing this information, which consists of several gravitational-wave cycles, we evolve only the outer portions of our grid beyond the time at which the code crashes in the center, effectively "escorting" the radiation out to the extraction radii.

To do this, we first stop the evolution shortly before it crashes, and we introduce a new spherical excision boundary that surrounds both black holes and has a radius of roughly 3 times the black-hole separation. This new excision boundary moves radially outward at slightly faster than the speed of light so that it is causally disconnected from the interior region where the code is crashing, and so that no boundary conditions are required on this boundary. We then continue the evolution on the truncated sphericalshell domain that extends from the new excision boundary to the outer boundary. To move both boundaries appropriately, we employ a new radial coordinate mapping

$$
r=A(t) r\left(r^{\prime}\right)+B(t)
$$

where $r\left(r^{\prime}\right)$ is given by Eq. (9). The functions $A(t)$ and $B(t)$ are chosen to satisfy three criteria: First, the inner boundary of the spherical shell moves outward with coordinate speed of unity, which turns out to be slightly superluminal. Second, the outer boundary location $R_{\text {bdry }}(t)$ has continuous first and second time derivatives at the time we transition to the truncated domain. And finally, the outer boundary location $R_{\text {bdry }}(t)$ approaches some fixed value at late times. The right panel of Fig. 2 shows the motion of the inner and outer radii for evolutions 30c-1 and 30c-2 (we did not perform wave escorting for 30c-3). For 30c-1, wave escorting extends the evolution for an additional time $220 \mathrm{~m}$ beyond the point at which the simulation stops in the center.

Figure 5 shows the gravitational waveform extracted at inertial coordinate radius $R=240 \mathrm{~m}$ for the run 30c-1. The brown vertical line indicates the time when wave escorting starts. Wave escorting allows us to extract another 4 cycles of gravitational waves. When computing the gravitationalwave strain $h(t)$ from the Newman-Penrose scalar $\Psi_{4}$ (see Eq. (11) below), one must choose integration constants during the time integration. These integration constants were chosen such that $h(t)$ has zero average and first moment [40], which is sufficiently accurate for the illustrative Fig. 5. To avoid errors caused by the choice of integration constants, the comparison to post-Newtonian waveforms below is based entirely on $\Psi_{4}$. 


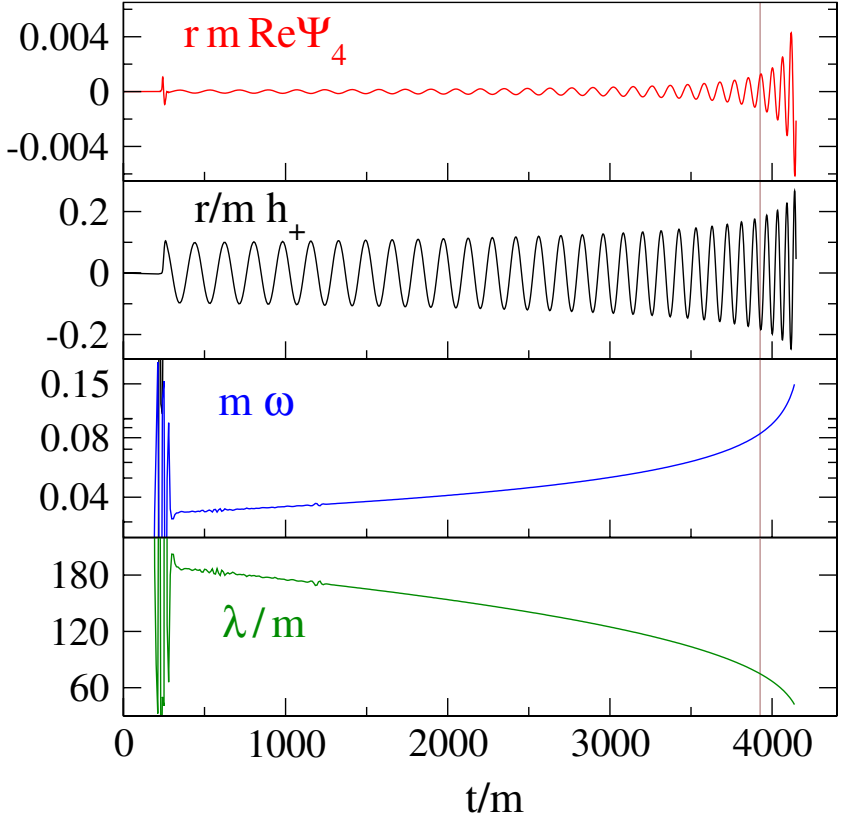

FIG. 5 (color online). Gravitational waveform extracted at $r=$ $240 \mathrm{~m}$. From top panel to bottom: The real part of the $(2,2)$ component of $r \Psi_{4}$; the gravitational-wave strain, obtained by two time integrals of $\operatorname{Re}\left(r \Psi_{4}\right)$; the frequency of the gravitational wave, Eq. (15); the gravitational wavelength, $\lambda=2 \pi / \omega$. The vertical brown line at $t \approx 3930 \mathrm{~m}$ indicates the time when "wave escorting" starts.

In the lower two panels of Fig. 5 there is a significant amount of noise near the beginning of the run, at $t<250 \mathrm{~m}$. This noise is barely evident in the top panel of Fig. 5 as well. The noise is a manifestation of "junk radiation," a pulse of radiation often seen at the beginning of numerical relativity simulations, and is caused by the initial data not being precisely a snapshot of an evolution that has been running for a long time. Among the effects that produce junk radiation are incorrect initial distortions of the individual holes, so that each hole radiates as it relaxes to its correct quasiequilibrium shape.

Our evolution code does not explicitly enforce either the Einstein constraints or the secondary constraints that arise from writing the system in first-order form. Therefore, examining how well these constraints are satisfied provides a useful consistency check. Figure 6 shows the constraint violations for run 30c-1. The top panel shows the $L^{2}$ norm of all the constraint fields of our first-order generalized harmonic system, normalized by the $L^{2}$ norm of the spatial gradients of the dynamical fields (see Eq. (71) of Ref. [69]). The bottom panel shows the same quantity, but without the normalization factor (i.e., just the numerator of Eq. (71) of Ref. [69]). The $L^{2}$ norms are taken over the entire computational volume that lies outside of apparent horizons. At early times, $t<500 \mathrm{~m}$, the constraints converge rather slowly with resolution because the junk radiation contains high frequencies. Convergence is more

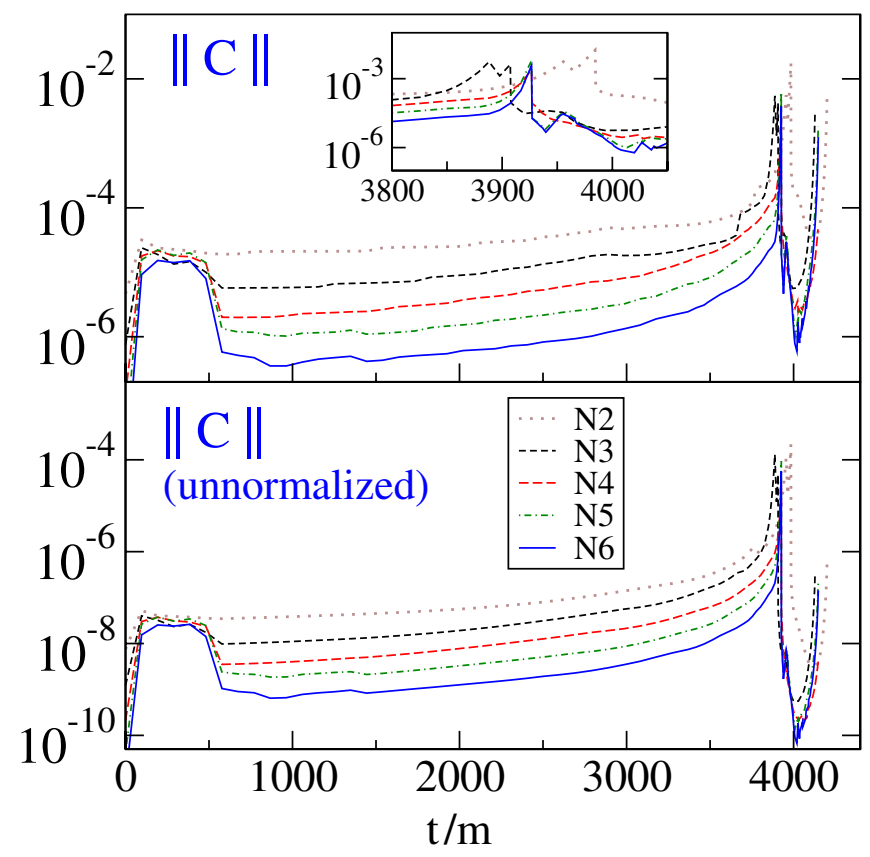

FIG. 6 (color online). Constraint violations of run 30c-1. The top panel shows the $L^{2}$ norm of all constraints, normalized by the $L^{2}$ norm of the spatial gradients of all dynamical fields. The bottom panel shows the same data, but without the normalization factor. Norms are taken only in the regions outside apparent horizons.

rapid during the smooth inspiral phase, after the junk radiation has exited through the outer boundary. The constraints increase around $t \sim 3900 \mathrm{~m}$ as the code begins to fail near the two merging holes, but then the constraints decrease again after the failing region is excised for wave escorting. The normalized constraint violations are less than $10^{-4}$ until just before the peak (which occurs at $t=$ $3930 \mathrm{~m}$ for all but the lowest resolutions). The size of the peak causes some concern that the waveforms at late times may be contaminated by constraint violations to a nonnegligible degree. However, near the peak, the constraint violations are large only in the inner regions of the domain near the black holes (note that the curves in Fig. 6 decrease by 2 orders of magnitude immediately after these inner regions are excised at $t=3930 \mathrm{~m}$ ). Because all constraint quantities propagate at the speed of light or slower for the formulation of Einstein's equations that we use, any influence that the constraint peak has on the extracted waveform occurs after the constraint violations have had time to propagate out to the wave-extraction zone. This is very late in the waveform, well after the gravitational-wave frequency reaches $m \omega=0.1$, as can be seen from the right panel of the spacetime diagram in Fig. 2.

\section{Waveform extraction}

Gravitational waves are extracted using the NewmanPenrose scalar $\Psi_{4}$, using the same procedure as in [40]. To summarize, given a spatial hypersurface with timelike unit 
normal $n^{\mu}$, and given a spatial unit vector $r^{\mu}$ in the direction of wave propagation, the standard definition of $\Psi_{4}$ is the following component of the Weyl curvature tensor,

$$
\Psi_{4}=-C_{\alpha \mu \beta \nu} \ell^{\mu} \ell^{\nu} \bar{m}^{\alpha} \bar{m}^{\beta},
$$

where $\ell^{\mu} \equiv \frac{1}{\sqrt{2}}\left(n^{\mu}-r^{\mu}\right)$, and $m^{\mu}$ is a complex null vector (satisfying $m^{\mu} \bar{m}_{\mu}=1$ ) that is orthogonal to $r^{\mu}$ and $n^{\mu}$. Here an overbar denotes complex conjugation.

For (perturbations of) flat spacetime, $\Psi_{4}$ is typically evaluated on coordinate spheres, and in this case the usual choices for $n^{\mu}, r^{\mu}$, and $m^{\mu}$ are

$$
\begin{aligned}
n^{\mu} & =\left(\frac{\partial}{\partial t}\right)^{\mu}, \\
r^{\mu} & =\left(\frac{\partial}{\partial r}\right)^{\mu}, \\
m^{\mu} & =\frac{1}{\sqrt{2} r}\left(\frac{\partial}{\partial \theta}+i \frac{1}{\sin \theta} \frac{\partial}{\partial \phi}\right)^{\mu},
\end{aligned}
$$

where $(r, \theta, \phi)$ denote the standard spherical coordinates. With this choice, $\Psi_{4}$ can be expanded in terms of spinweighted spherical harmonics of weight -2 :

$$
\Psi_{4}(t, r, \theta, \phi)=\sum_{l m} \Psi_{4}^{l m}(t, r)_{-2} Y_{l m}(\theta, \phi),
$$

where the $\Psi_{4}^{l m}$ are expansion coefficients defined by this equation.

For curved spacetime, there is considerable freedom in the choice of the vectors $r^{\mu}$ and $m^{\mu}$, and different researchers have made different choices $[8,32,95-99]$ that are all equivalent in the $r \rightarrow \infty$ limit. We choose these vectors by first picking an extraction two-surface $\mathcal{E}$ that is a coordinate sphere $\left(r^{2}=x^{2}+y^{2}+z^{2}\right.$ using the global asymptotically Cartesian coordinates employed in our code) centered on the center of mass of the binary system, i.e. the point of symmetry. We choose $r^{\mu}$ to be the outward-pointing spatial unit normal to $\mathcal{E}$ (that is, we choose $r_{i}$ proportional to $\nabla_{i} r$ and raise the index with the spatial metric). Then we choose $m^{\mu}$ according to Eq. (12c), using the standard spherical coordinates $\theta$ and $\phi$ defined on these coordinate spheres. Finally we use Eqs. (11) and (13) to define the $\Psi_{4}^{l m}$ coefficients.

Note that the $m^{\mu}$ vector used here is not exactly null nor exactly of unit magnitude at finite $r$. The resulting $\Psi_{4}^{l m}$ at finite $r$ will disagree with the waveforms observed at infinity. Our definition does, however, agree with the standard definition given in Eqs. (11)-(13) as $r \rightarrow \infty$. Because we extrapolate the extracted waves to find the asymptotic radiation field (see Sec. II F), these effects should not play a role in our PN comparisons: Relative errors in $\Psi_{4}^{l m}$ introduced by using the simple coordinate tetrad fall off like $1 / r$, and thus should vanish after extrapolating to obtain the asymptotic behavior. While more careful treatment of the extraction method- such as those discussed in [100-
102] - may improve the quality of extrapolation and would be interesting to explore in the future, the naive choice made here should be sufficient to ensure that the waveform after extrapolation is correct to the accuracy needed for these simulations.

In this paper, we focus on the $(l, m)=(2,2)$ mode. Following common practice (see e.g. $[8,14]$ ), we split the extracted waveform into real phase $\phi$ and real amplitude $A$, defined by

$$
\Psi_{4}^{22}(r, t)=A(r, t) e^{-i \phi(r, t)} .
$$

The gravitational-wave frequency is given by

$$
\omega=\frac{d \phi}{d t} .
$$

The minus sign in the definition of $\phi$ is chosen so that the phase increases in time and $\omega$ is positive. Equation (14) defines $\phi$ only up to multiples of $2 \pi$. These multiples of $2 \pi$ are chosen to make $\phi$ continuous through each evolution, still leaving an overall multiple of $2 \pi$ undetermined. We will consider only phase differences in this paper, so the choice of this overall phase offset is irrelevant.

\section{E. Convergence of extracted waveforms}

In this section we examine the convergence of the gravitational waveforms extracted at fixed radius, without extrapolation to infinity. This allows us to study the behavior of our code without the complications of extrapolation. The extrapolation process and the resulting extrapolated waveforms are discussed in Sec. IIF.

The top panel of Fig. 7 shows the convergence of the gravitational-wave phase $\phi$ with numerical resolution for the run $30 \mathrm{c}-1$. For this plot, the waveform is extracted at a fixed radius $R=77 \mathrm{~m}$. Each line shows the difference between $\phi$ computed at some particular resolution and $\phi$ computed from our highest-resolution run 30c-1/N6. When subtracting results at different resolutions, no time or phase adjustment has been performed. The difference in $\phi$ between the two highest-resolution runs is smaller than 0.03 radians throughout the run, and it is smaller than 0.02 radians between $t=1000 m$ and the point at which $m \omega=$ 0.1 .

At times before $1000 \mathrm{~m}$, the phase convergence of our simulation is limited to about 0.05 radians because of effects of junk radiation (described at the end of Sec. II C). The sharp pulse of junk radiation has comparatively large numerical truncation error, and excites all characteristic modes at truncation-error level, including waves that propagate back toward the origin. Generation of these secondary waves stops when the pulse of junk radiation leaves through the outer boundary (i.e., after one light-crossing time). Because we use the improved outer boundary conditions of Rinne et al. [74], there are no significant reflections when the junk radiation passes through the outer boundary. However, the waves produced 


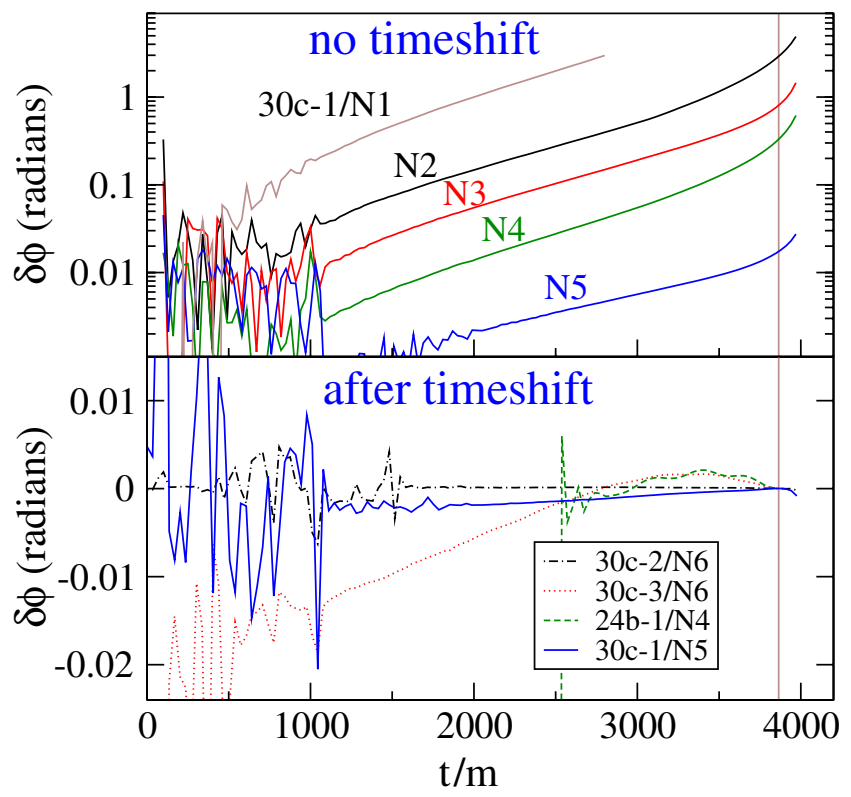

FIG. 7 (color online). Convergence of the gravitational-wave phase extracted at radius $R=77 \mathrm{~m}$. All lines show differences with respect to our highest-resolution run, 30c-1/N6. The top panel shows different resolutions of the same run 30c-1; no time or phase shifts have been performed. The bottom panel compares different runs, aligning the runs at $m \omega=0.1$ by a time and phase shift. The thin vertical line indicates the time at which $m \omega=0.1$ for $30 \mathrm{c}-1 / \mathrm{N} 6$.

before the junk radiation leaves remain in the computational domain for two additional light-crossing times, until they eventually leave through the outer boundary.

The bottom panel of Fig. 7 shows phase comparisons between different waveforms after we perform a time shift and phase shift so that the waveforms agree at $m \omega=0.1$. Our procedure for time shifting and phase shifting is the same as the shifting procedure we use to compare NR with PN waveforms (see Sec. IV B), so that the error estimates we extract from the bottom panel of Fig. 7 are relevant for our later NR-PN comparison.

There are three different types of comparisons shown in the bottom panel of Fig. 7: Phase differences between runs with the same initial data but with different outer boundary locations, phase differences between runs with different initial data, and phase differences between different numerical resolutions of the same run (this last comparison is the same as what is shown in the top panel, except in the bottom panel the waveforms are time and phase shifted). We will discuss all three of these in turn.

First, we compare the phase difference of 30c-1/N6 with runs that have different outer boundary locations. Run 30c2 (with more distant outer boundary) agrees to within 0.002 radians with run $30 \mathrm{c}-1$, but run $30 \mathrm{c}-3$ (with closer outer boundary), has a much larger phase difference with 30c-1. We believe that this is because run $30 \mathrm{c}-3$ has a very small ratio of outer boundary location to gravitational wave- length: $R / \lambda$ is about 1.1 for the first two-thirds of the run, and remains less than 2 for the entire run.

We can explain the order of magnitude of these phase differences using the analysis of Buchman and Sarbach [82]. Our outer boundary conditions are not perfectly absorbing, but instead they reflect some fraction of the outgoing radiation. ${ }^{5}$ The ratio of the amplitude of curvature perturbations (i.e. $\Psi_{4}$ ) of the reflected wave to that of the outgoing wave is

$$
q \approx \frac{3}{2(2 \pi)^{4}}\left(\frac{\lambda}{R}\right)^{4}
$$

The incoming reflected waves grow like $1 / r$ as they travel inward just like the outgoing waves decrease by $1 / r$ as they propagate outward. Therefore, the ratio of amplitudes of incoming and outgoing waves will have approximately the same value, $q$, at smaller radii, and we assume for the sake of this rough argument that this ratio remains equal to $q$ even in the vicinity of the black holes (where it is no longer technically meaningful to talk about "radiation"). Now consider the second time derivative of the gravitationalwave phase, $\ddot{\phi}$; this is nonzero only because of gravitational-wave emission, so $\ddot{\phi}$ is proportional to some power of the outgoing wave amplitude. To get the correct power, we can use Eq. (47) to find $\dot{x} \sim x^{5}$, so Eq. (38) yields $\ddot{\phi} \sim x^{11 / 2}$ (we assume gravitational-wave phase is twice the orbital phase). The amplitude of $\Psi_{4}$ scales like $x^{4}$, so $\ddot{\phi} \sim A^{11 / 8}$. Let us assume for the sake of this rough error estimate that the change in $\ddot{\phi}$ due to the ingoing reflected wave scales similarly with amplitude, $\ddot{\phi} \sim \bar{A}^{11 / 8}$, where $\bar{A}=q A$ is the amplitude of the reflected ingoing wave. Therefore the unphysical gravitational-wave force acting back on the system due to boundary reflections will cause fractional errors in the second derivative of the phase of about $q^{11 / 8}$. That is, the error $\delta \phi$ caused by the improper boundary condition will be given by

$$
\frac{d^{2} \delta \phi}{d t^{2}}=q^{11 / 8} \frac{d^{2} \phi}{d t^{2}}
$$

Integrating this yields $\delta \phi=q^{11 / 8} \phi$, where $\phi$ is the total gravitational-wave phase accumulated during the evolution. For 30c-3, $\lambda / R \sim 0.9$, so $q \sim 6 \times 10^{-4}$, which yields $\delta \phi \sim 0.08$ radians for an accumulated gravitational-wave phase of about 200 radians. This rough estimate agrees in order of magnitude with the phase difference between 30c3 and 30c-1 as shown in the bottom panel of Fig. 7. The run $30 \mathrm{c}-1$ has an outer boundary about 2.5 farther away, reducing the reflection coefficient by a factor $2.5^{4} \approx 40$, so for 30c-1 this estimate of the phase error gives $\delta \phi=5 \times$ $10^{-4}$ radians. Therefore, we expect reflection of the out-

\footnotetext{
${ }^{5}$ However, in a comparison of various boundary conditions [74], the boundary conditions we use produced smaller reflections than other boundary conditions commonly used in numerical relativity.
} 
going radiation at the outer boundary to be insignificant for $30 \mathrm{c}-1$. This is confirmed by the excellent agreement between runs 30c-1 and 30c-2 (the latter having even larger outer boundary).

The second comparison shown in the lower panel of Fig. 7 is the phase difference between 30c-1/N6 and 24b$1 / \mathrm{N} 4$, a shorter 8 -orbit evolution started from a separate initial data set (set $24 \mathrm{~b}$ in Table I) with a separate eccentricity-reduction procedure. The phase agreement between these two runs (including an overall time shift and phase shift) is better than 0.01 radians for a total accumulated phase of $\sim 100$ radians of the 8 -orbit run, i.e. better than one part in $10^{4}$. Run $24 \mathrm{~b}-1$ has a similar outer boundary location as run 30c-3, and indeed both of these runs show similar phase differences from 30c-1.

Finally, the third comparison shown in the lower panel of Fig. 7 is the phase difference between the two highest resolutions of the run 30c-1 when a time shift is applied. For $t \gtrsim 1000 m$ the agreement is much better than without the time shift (see upper panel), indicating that the dominant error is a small difference in the overall evolution time. For the post-Newtonian comparisons we perform in the second part of this paper, waveforms are always aligned at specific frequencies by applying time and phase shifts. Therefore, the time-shifted phase difference as displayed in the lower panel is the most appropriate measure of numerical truncation error for these PN comparisons. This difference is less than 0.003 radians after $t=1000 \mathrm{~m}$ but is larger, about 0.02 radians, at early times where the waveforms are noisy because of junk radiation.

We now compare the gravitational-wave amplitudes of different runs in the same manner as we compared the gravitational-wave phases. Figure 8 presents convergence data for the amplitude of the gravitational waves for the same runs as shown in Fig. 7. Spatial truncation error for the amplitude is less than $0.1 \%$ for $t / m>1000$, and earlier than this it is limited by residual noise from the junk radiation. Differences (including time shifts) between runs of different lengths are shown in the lower panel of Fig. 8. These differences are even smaller, but because of their small size, they are dominated by noise for about the first half of the run. The oscillations apparent in the comparison to $24 \mathrm{~b}-1$ are caused by the larger orbital eccentricity of $24 b-1$ (cf. Table I).

\section{F. Extrapolation to infinity}

The quantity of interest to gravitational-wave detectors is the gravitational waveform as seen by an observer effectively infinitely far from the source. Our numerical simulations, in contrast, cover only a region of finite volume around the source, and our numerical waveforms are extracted at a finite radius. Waveforms extracted at a finite radius can differ from those extracted at infinity because of effects discussed in Sec. IID; these effects can lead to phase errors of several tenths of a radian and relative

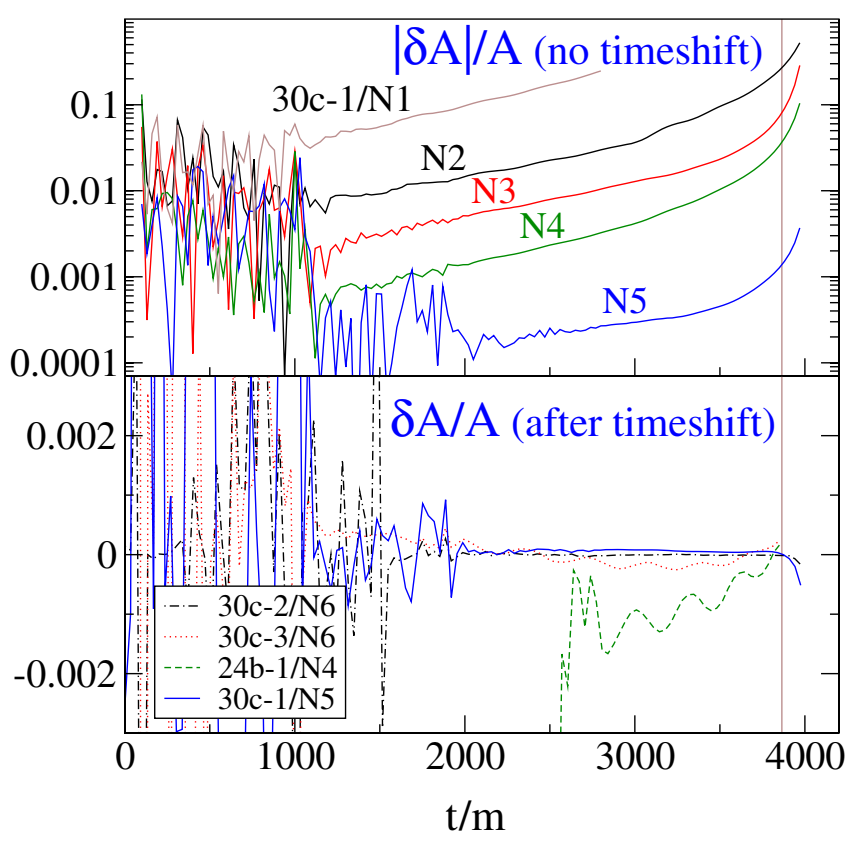

FIG. 8 (color online). Convergence of the gravitational-wave amplitude extracted at radius $R=77 \mathrm{~m}$. This plot corresponds to Fig. 7, except that relative amplitude differences are shown. The thin vertical line indicates the time at which $m \omega=0.1$ for $30 \mathrm{c}-1 /$ N6.

amplitude errors of several percent. To avoid such errors we extrapolate to infinite extraction radius as follows.

We extract data for $\Psi_{4}$ on coordinate spheres of coordinate radii $r / m=75,80,85, \ldots, 240$, as described in Sec. II D. These extracted waveforms are shifted in time relative to one another because of the finite light-travel time between these extraction surfaces. We correct for this by shifting each waveform by the tortoise-coordinate radius at that extraction point [95]

$$
r^{*}=r_{\text {areal }}+2 M_{\mathrm{ADM}} \ln \left(\frac{r_{\text {areal }}}{2 M_{\mathrm{ADM}}}-1\right) .
$$

Here $M_{\mathrm{ADM}}$ is the ADM mass of the initial data, and $r_{\text {areal }}=\sqrt{A / 4} \bar{\pi}$, where $A$ is the area of the extraction sphere. This is not the only possible choice for the retarded time - for example, the waveforms could be shifted so that the maxima of the amplitude align [41]. It has also been suggested [103] that the time shift should change with the amount of radiated energy - essentially, that the factor of $M_{\text {ADM }}$ should be replaced by the amount of mass interior to the extraction radius at each time. We leave investigation of other choices of retarded time for future work.

Figure 9 presents the areal radius during the evolution at several typical extraction radii. The areal radius of these extraction surfaces is constant to within about $0.01 \mathrm{~m}$, and to the same precision, $r_{\text {areal }}=r+M_{\mathrm{ADM}}$. This relationship is not surprising, because the initial data is conformally flat, so that for coordinate spheres $r_{\text {areal }}=$ $r+M_{\mathrm{ADM}}+\mathcal{O}\left(M_{\mathrm{ADM}} / r\right)$. For convenience, we simply set 


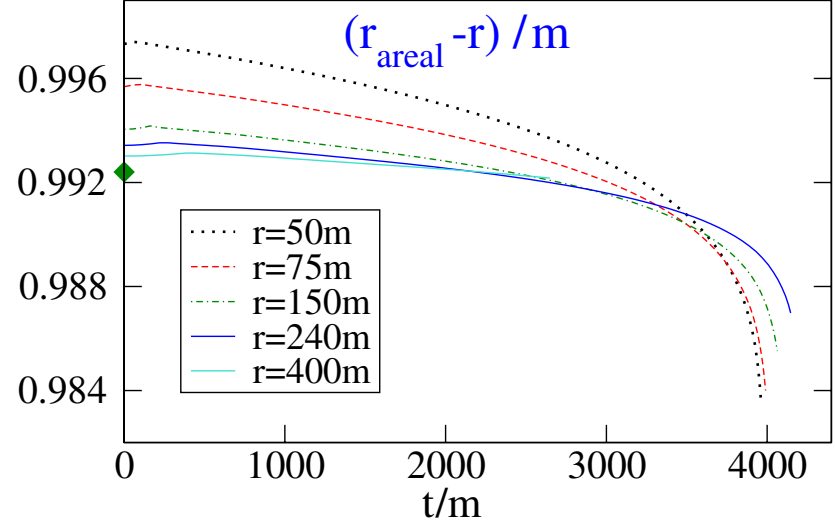

FIG. 9 (color online). Difference between areal radius $r_{\text {areal }}$ and coordinate radius $r$ of selected extraction surfaces. $r_{\text {areal }}$ remains constant to within $0.01 \mathrm{~m}$ during the evolution. The diamond indicates $M_{\mathrm{ADM}} / m$ of the initial data.

$r_{\text {areal }}=r+M_{\mathrm{ADM}}$ in Eq. (18), rather than explicitly integrating to find the area of each extraction sphere.

After the time shift, each waveform is a function of retarded time, $t-r^{*}$. At a given value of retarded time, we have a series of data points - one for each extraction radius. We fit phase and amplitude of these data separately to a polynomial in $1 / r$,

$$
\begin{aligned}
& \phi\left(t-r^{*}, r\right)=\phi_{(0)}\left(t-r^{*}\right)+\sum_{k=1}^{n} \frac{\phi_{(k)}\left(t-r^{*}\right)}{r^{k}}, \\
& r A\left(t-r^{*}, r\right)=A_{(0)}\left(t-r^{*}\right)+\sum_{k=1}^{n} \frac{A_{(k)}\left(t-r^{*}\right)}{r^{k}} .
\end{aligned}
$$

The leading-order term of each polynomial, as a function of retarded time, is then the desired asymptotic waveform:

$$
\begin{aligned}
& \phi\left(t-r^{*}\right)=\phi_{(0)}\left(t-r^{*}\right), \\
& r A\left(t-r^{*}\right)=A_{(0)}\left(t-r^{*}\right) .
\end{aligned}
$$

We find good convergence of this method as we increase the order $n$ of the extrapolating polynomial. Figure 10 shows the difference in phase between waveforms extrapolated using successively higher-order polynomials. We see a broad improvement in the accuracy of the phase with increasing order, but unfortunately, higher-order extrapolations tend to amplify the noise. Our preferred choice is $n=3$ extrapolation, resulting in extrapolation errors of $\lesssim$ 0.003 radians for $t-r^{*} \gtrsim 1000 m$.

Figure 11 is analogous to Fig. 10, except that it shows relative differences in the extrapolated amplitudes. The basic picture agrees with the phase extrapolation: Higherorder extrapolation reduces the errors, but amplifies noise. Our preferred choice $n=3$ gives a relative amplitude error of $\lesssim 0.002$ for $t-r^{*} \geq 1000 m$, dropping to less than 0.001 for $t-r^{*} \gtrsim 2000 m$.

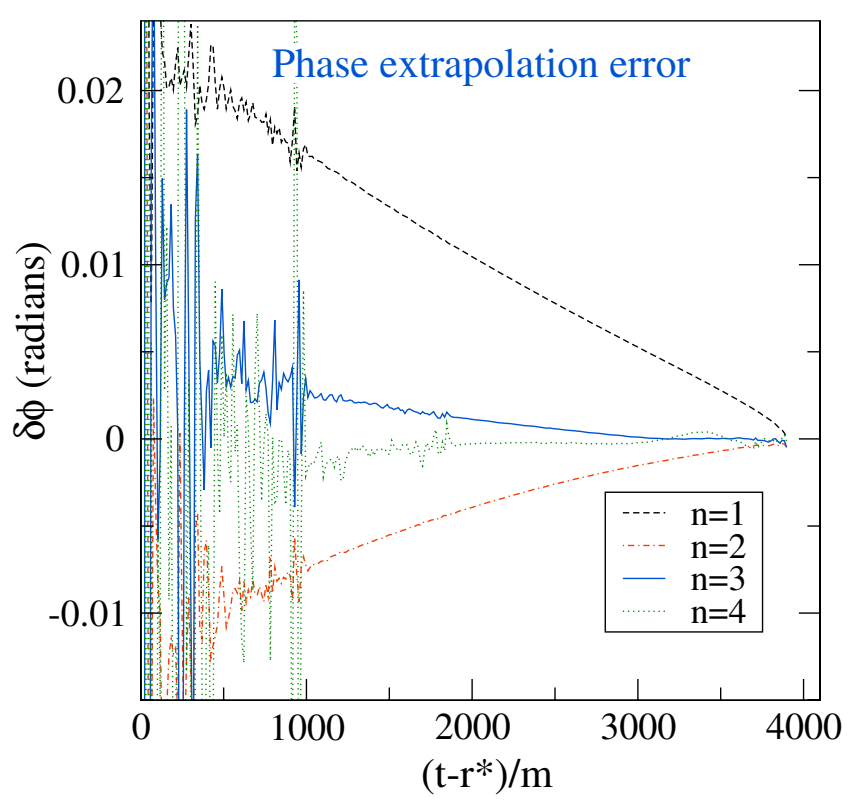

FIG. 10 (color online). Error of phase extrapolation to infinity for extrapolation of order $n$, cf. Eq. (19). Plotted are absolute differences between extrapolation with order $n$ and $n+1$. Increasing the order of the polynomial increases accuracy, but also amplifies noise.

Phase and amplitude extrapolation become increasingly more accurate at late times. The main obstacle to accuracy seems to be near-zone effects scaling with powers of $(\lambda / r)$, where $\lambda$ is the wavelength of the gravitational wave. The

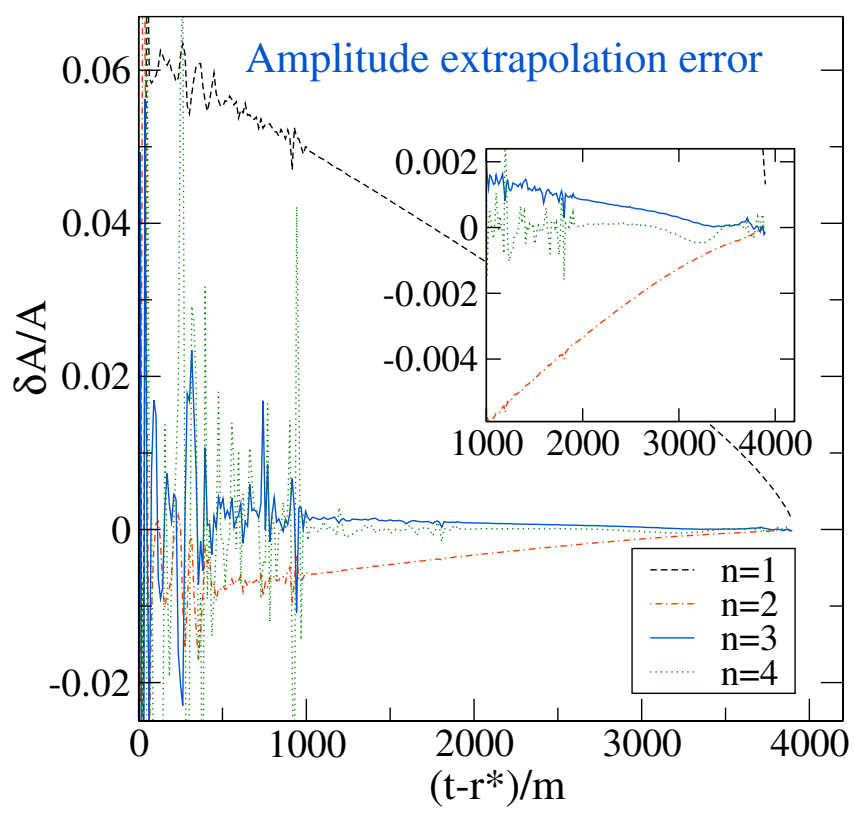

FIG. 11 (color online). Error of amplitude extrapolation to infinity for extrapolation with order $n$, cf. Eq. (20). Plotted are relative amplitude differences between extrapolation with orders $n$ and $n+1$. The inset is an enlargement for $t-r^{*} \geq 1000 m$. 
wavelength is quite large at the beginning of the simulation $(\lambda \approx 180 \mathrm{~m}$, cf. Fig. 5), but becomes shorter during the evolution, so that even low-order extrapolation is quite accurate at late times. Alternatively, near-zone effects can be mitigated by using data extracted at large values of $r$. It is precisely because of these near-zone effects that we have chosen to ignore data extracted at $r<75 \mathrm{~m}$ when we extrapolate to infinity.

In Figs. 12 and 13, we show the effects of extrapolation using different ranges of extracted data. Using data extracted every $5 m$ in the range $r=50 m-90 m$ results in noticeable differences early in the run-though it is adequate later in the run. For ranges at higher radii (e.g. $[75 m, 150 m]$ or $[150 m, 240 m])$, the accuracy is not highly variable, though we find that noise is increased when using data from such a smaller range of extraction radii.

To estimate the errors generated by not extrapolating waveforms to infinity at all, Fig. 12 contains also the phase difference between wave extraction at two finite radii $(90 \mathrm{~m}$ and $240 \mathrm{~m}$ ) and our preferred extrapolated phase at infinity. The dotted lines show such phase differences when only a time shift by the tortoise-coordinate radius of the extraction sphere is applied. The errors are dramatic, tenths of radians or more, even very late in the run. When matching to post-Newtonian waveforms, we are free to add an overall time and phase shift (cf. Sec. IV B). Therefore, the

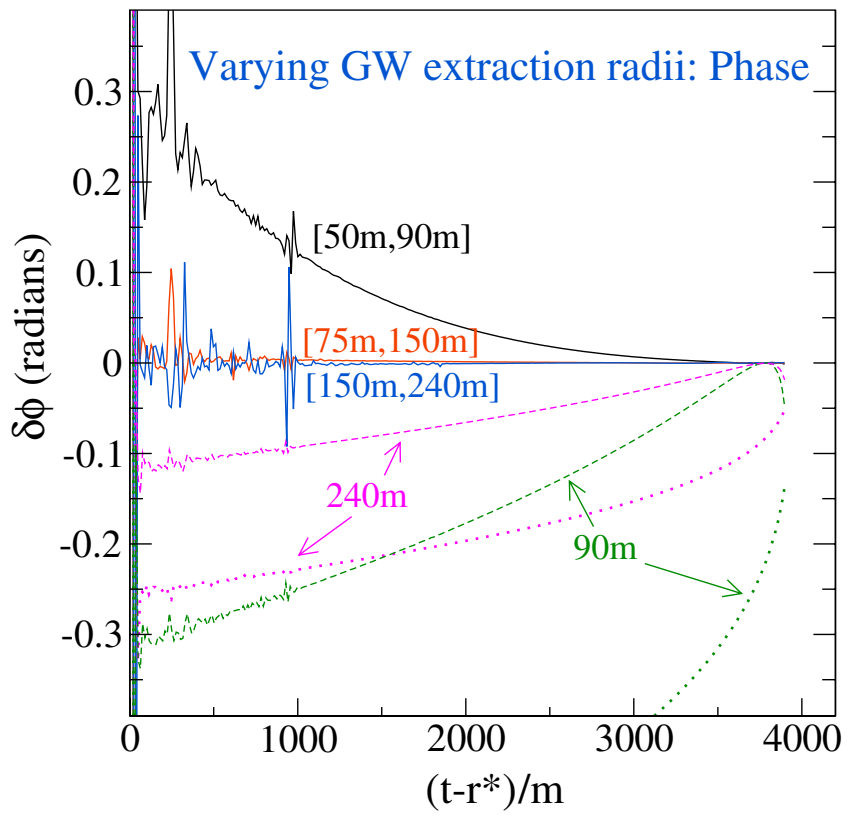

FIG. 12 (color online). Effect of wave-extraction radii on extrapolated phase. Each curve represents the difference from our preferred wave extrapolation using $r \in[75 \mathrm{~m}, 240 \mathrm{~m}]$. The three solid curves represent extrapolation from different intervals of extraction radii. The curves labeled " $240 m$ " and " $90 m$ " represent differences from waves extracted at these two radii, without any extrapolation, for two cases: time and phase shifted so that $\phi$ and $\dot{\phi}$ match at $m \omega=0.1$ (dashed line), and without these shifts (dotted line).

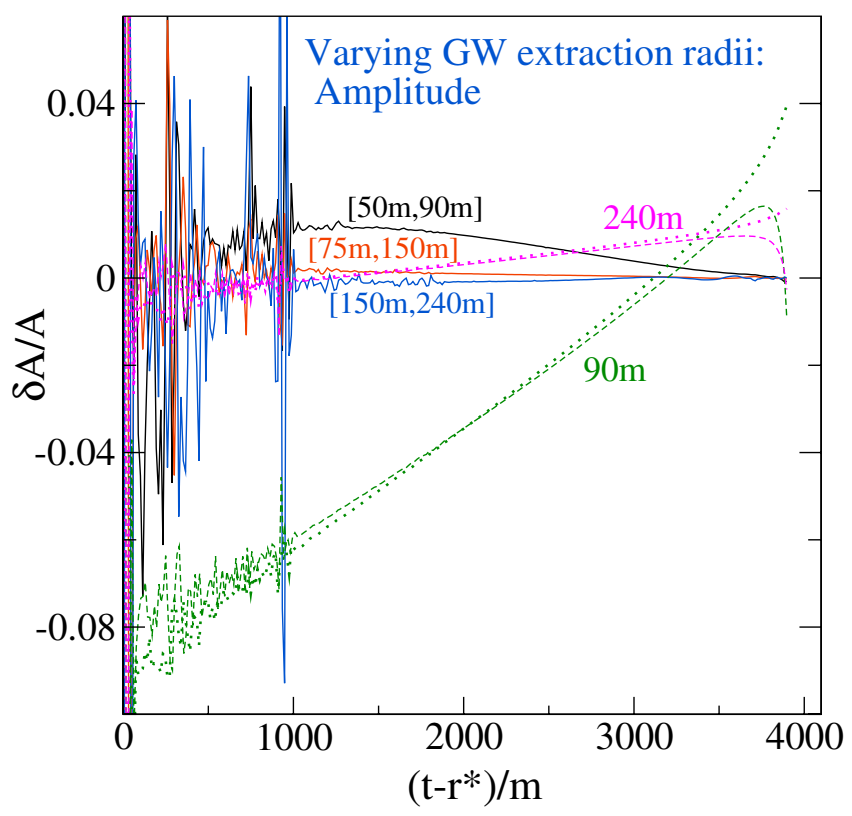

FIG. 13 (color online). Effect of choice of wave-extraction radii on extrapolated amplitude. Each curve represents the (relative) amplitude difference to our preferred wave extrapolation using $r \in[75 \mathrm{~m}, 240 \mathrm{~m}]$. The three solid curves represent extrapolation from different intervals of extraction radii. The curves labeled " $240 m$ " and " $90 m$ " represent differences from waves extracted at these two radii, without any extrapolation, for two cases: time and phase shifted so that $\phi$ and $\dot{\phi}$ match at $m \omega=0.1$ (dashed line), and without these shifts (dotted line).

dashed lines in Fig. 12 show phase differences with the same unextrapolated waveforms as shown by the dotted lines, except that a phase and time shift has been applied so that the $\phi$ and $\dot{\phi}$ agree with those of the extrapolated waveform late in the run (where $m \omega=0.1$ ), where the wavelengths are shortest and wave extraction is expected to work best. Even with such an adjustment, the gravitational-wave phase extracted at $r=90 \mathrm{~m}$ differs by about $0.1 \mathrm{rad}$ at $t \sim 1000 \mathrm{~m}$ before coalescence, with this difference growing to 0.3 radians at the start of our simulation.

Figure 13 makes the same comparison for the gravitational-wave amplitude. Wave extraction at $r=$ $90 m$ results in relative amplitude errors of up to 8 percent, and of about 2 percent even in the last $1000 \mathrm{~m}$ of our simulation. We also point out that the errors due to finite extraction radius decay approximately as the inverse of the extraction radius: For waves extracted at $r=240 \mathrm{~m}$ the errors are smaller than for waves extracted at $r=90 m$ by about a factor of 3, as can be seen in Figs. 12 and 13; for wave extraction at $r=45 \mathrm{~m}$, the errors would be approximately twice as large as the $r=90 \mathrm{~m}$ case. The errors introduced by using a finite extraction radius are significantly larger than our truncation error (even at extraction radius $240 m$ ). Therefore extrapolation to infinity is essential to realize the full accuracy of our simulations. 


\section{G. Estimated time of merger}

Since we have not yet been successful with simulating the merger, we do not precisely know when merger occurs. However, by comparing the orbital and gravitational-wave frequencies to already published results, we can nevertheless estimate the time of merger.

The simulation presented in Fig. 3 stops at time $t=$ $3929 m$ when the horizons of the black holes become too distorted just before merger. At that point, the proper separation between the horizons is $\sim 4.0 \mathrm{~m}$, and the orbital frequency has reached $m \Omega_{\text {orbit }}=0.125$; comparison with [32] suggests this is about $15 \mathrm{~m}$ before formation of a common apparent horizon, i.e. the common horizon should form in our simulations at $t_{\mathrm{CAH}} \approx 3945 \mathrm{~m}$.

The waveform extrapolated to infinity ends at $t-r^{*}=$ $3897 m$ at a gravitational-wave frequency of $m \omega \approx 0.16$. This places the end of the waveform at about $50 \mathrm{~m}$ (or $\sim 1.5$ cycles) before formation of a common apparent horizon ${ }^{6}$ (judged by comparison with [32]). Thus, we estimate the formation of a common horizon to correspond to a retarded time of approximately $\left(t-r^{*}\right)_{\mathrm{CAH}} \approx 3950 \mathrm{~m}$.

\section{GENERATION OF POST-NEWTONIAN WAVEFORMS}

It is not our intention to review all of PN theory, but to summarize the important points that go into the construction of the post-Newtonian waveforms that we will compare to our numerical simulation. For a complete review of post-Newtonian methods applied to inspiralling compact binaries, see the review article by Blanchet [104].

The post-Newtonian approximation is a slow-motion, weak-field approximation to general relativity with an expansion parameter $\epsilon \sim(v / c)^{2} \sim\left(G m / r c^{2}\right)$. For a binary system of two point masses $m_{1}$ and $m_{2}, v$ is the magnitude of the relative velocity, $m$ is the total mass, and $r$ is the separation. In order to produce a post-Newtonian waveform, it is necessary to solve both the post-Newtonian equations of motion describing the binary, and the postNewtonian equations describing the generation of gravitational waves.

Solving the equations of motion yields explicit expressions for the accelerations of each body in terms of the positions and velocities of the two bodies [105-115]. The two-body equations of motion can then be reduced to relative equations of motion in the center-of-mass frame in terms of the relative position and velocity [116]. The relative acceleration is currently known through 3.5PN order, where 0PN order for the equations of motion corresponds to Newtonian gravity. The effects of radiation

\footnotetext{
${ }^{6}$ The waveform ends somewhat further from merger than the orbital trajectory, because the artificial boundary is placed initially at a radius $\sim 15 \mathrm{~m}$, and then moves outward somewhat faster than the speed of light, thus overtaking the very last part of the waveform as it travels to the wave-extraction radii.
}

reaction (due to the emission of gravitational waves) enters the relative acceleration starting at $2.5 \mathrm{PN}$ order. The relativistic corrections to the relative acceleration at $1 \mathrm{PN}, 2 \mathrm{PN}$, and 3PN order (ignoring the radiation reaction terms at 2.5PN and 3.5PN order) admit a conserved center-of-mass binding energy through 3PN order [117]. There is no $2.5 \mathrm{PN}$ or 3.5PN order contribution to the energy.

Solving the post-Newtonian wave generation problem yields expressions for the gravitational waveform $h_{i j}$ and gravitational-wave flux $\mathcal{L}$ in terms of radiative multipole moments [118]. These radiative multipole moments are in turn related to the source multipole moments, which can be given in terms of the relative position and relative velocity of the binary [119]. For the gravitational-wave generation problem, PN orders are named with respect to the leadingorder waveform and flux, which are given by the quadrupole formalism. Thus, for example, $1.5 \mathrm{PN}$ order in the wave generation problem represents terms of order $(v / c)^{3}$ beyond quadrupole. Higher-order effects enter both through post-Newtonian corrections to the mass quadrupole, as well as effects due to higher multipole moments. Starting at $1.5 \mathrm{PN}$ order the radiative multipole moments include nonlinear and noninstantaneous (i.e. depend upon the past history of the binary) interactions among the source multipole moments (e.g. gravitational-wave tails) [119-122].

It was recognized early that simply plugging in the orbital evolution predicted by the equations of motion into the expressions for the waveform would not generate templates accurate enough for matched filtering in detecting gravitational waves [123]. This is because radiation reaction enters the equations of motion only at the 2.5PN order; hence computing a waveform to $k \mathrm{PN}$ order beyond the quadrupole formalism would require $2.5+k \mathrm{PN}$ orders in the equations of motion. In order to obtain as accurate a post-Newtonian waveform as possible it is thus necessary to introduce the assumption of an adiabatic inspiral of a quasicircular orbit, as well as the assumption of energy balance between the orbital binding energy and the energy emitted by the gravitational waves.

\section{A. Adiabatic inspiral of quasicircular orbits}

The emission of gravitational radiation causes the orbits of an isolated binary system to circularize [50]. Thus it is a reasonable assumption to model the orbital evolution of the binary as a slow adiabatic inspiral of a quasicircular orbit. With this assumption, post-Newtonian expressions for the orbital energy $E$ and gravitational energy flux $\mathcal{L}$ are currently known through 3.5PN order [124-128]. These expressions can be given in terms of a parameter related to either the harmonic coordinate separation $r$, or to the orbital frequency $\Omega$. We choose to use the expressions given in terms of a frequency-related parameter

$$
x \equiv\left(\frac{G m \Omega}{c^{3}}\right)^{2 / 3}
$$


rather than a coordinate-related parameter, because the coordinate relationship between the numerical simulation and the harmonic coordinates used in post-Newtonian approximations is unknown. The orbital energy for an equal-mass system is given by [104]

$$
\begin{aligned}
E= & -\frac{m c^{2}}{8} x\left[1-\frac{37}{48} x-\frac{1069}{384} x^{2}\right. \\
& \left.+\left(\frac{1427365}{331776}-\frac{205}{384} \pi^{2}\right) x^{3}\right],
\end{aligned}
$$

and the gravitational-wave flux for an equal-mass system is given by [104]

$$
\begin{aligned}
\mathcal{L}= & \frac{2 c^{5}}{5 G} x^{5}\left\{1-\frac{373}{84} x+4 \pi x^{3 / 2}-\frac{59}{567} x^{2}-\frac{767}{42} \pi x^{5 / 2}\right. \\
& +\left[\frac{18608019757}{209563200}+\frac{355}{64} \pi^{2}-\frac{1712}{105} \gamma\right. \\
& \left.\left.-\frac{856}{105} \ln (16 x)\right] x^{3}+\frac{16655}{6048} \pi x^{7 / 2}\right\}
\end{aligned}
$$

where $\gamma=0.577216 \ldots$ is Euler's constant.

\section{B. Polarization waveforms}

The gravitational polarization waveforms for a quasicircular orbit in the $x-y$ plane, as measured by an observer at spherical coordinates $(R, \hat{\theta}, \hat{\phi})$, are given by

$$
\begin{gathered}
h_{+}=\frac{2 G \mu}{c^{2} R} x\{-(1+\cos \hat{\theta}) \cos 2(\Phi-\hat{\phi})+\cdots\}, \\
h_{\times}=\frac{2 G \mu}{c^{2} R} x\{-2 \cos \hat{\theta} \sin 2(\Phi-\hat{\phi})+\cdots\},
\end{gathered}
$$

where $\Phi$ is the orbital phase (measured from the $x$-axis) and $\mu=m_{1} m_{2} / m$ is the reduced mass. The polarization waveforms are currently known through $2.5 \mathrm{PN}$ order $[129,130]$.

\section{Optimally oriented observer}

For an equal-mass binary the polarization waveforms along the $z$-axis (i.e. the optimally oriented observer along the normal to the orbital plane) are given by $[129,130]$

$$
\begin{aligned}
h_{+}^{(z)}= & \frac{G m}{2 c^{2} R} x\left(\operatorname { c o s } 2 \Phi \left\{-2+\frac{17}{4} x-4 \pi x^{3 / 2}+\frac{15917}{2880} x^{2}\right.\right. \\
& \left.+9 \pi x^{5 / 2}\right\}+\sin 2 \Phi\left\{-12 \ln \left(\frac{x}{x_{0}}\right) x^{3 / 2}\right. \\
& \left.\left.+\left[\frac{59}{5}+27 \ln \left(\frac{x}{x_{0}}\right)\right] x^{5 / 2}\right\}\right),
\end{aligned}
$$

$$
\begin{aligned}
h_{\times}^{(z)}= & \frac{G m}{2 c^{2} R} x\left(\operatorname { s i n } 2 \Phi \left\{-2+\frac{17}{4} x-4 \pi x^{3 / 2}+\frac{15917}{2880} x^{2}\right.\right. \\
& \left.+9 \pi x^{5 / 2}\right\}+\cos 2 \Phi\left\{12 \ln \left(\frac{x}{x_{0}}\right) x^{3 / 2}\right. \\
& \left.\left.-\left[\frac{59}{5}+27 \ln \left(\frac{x}{x_{0}}\right)\right] x^{5 / 2}\right\}\right),
\end{aligned}
$$

where

$$
\ln x_{0} \equiv \frac{11}{18}-\frac{2}{3} \gamma+\frac{2}{3} \ln \left(\frac{G m}{4 b c^{3}}\right)
$$

is a constant frequency scale that depends upon the constant time scale $b$ entering the gravitational-wave tail contribution to the polarization waveforms [131,132]. The freely specifiable constant $b$ corresponds to a choice of the origin of radiative time $T$ with respect to harmonic time $t$, and enters the relation between the retarded time $T_{R}=$ $T-R / c$ in radiative coordinates (the coordinates in which the waveform is given) and the retarded time $t-r / c$ in harmonic coordinates (the coordinates in which the equations of motion are given) [131,132]:

$$
T_{R}=t-\frac{r}{c}-\frac{2 G M_{\mathrm{ADM}}}{c^{3}} \ln \left(\frac{r}{b c}\right) .
$$

Here $M_{\mathrm{ADM}}$ is the ADM mass (mass monopole) of the binary system.

\section{The (2, 2) mode}

When comparing a post-Newtonian waveform with data from a physical gravitational-wave detector, it is necessary to compare waves emitted in a certain direction $(\hat{\theta}, \hat{\phi})$ with respect to the source. However, comparing waveforms between PN and numerical simulations can be done in all directions simultaneously by decomposing the waveforms in terms of spherical harmonics and then comparing different spherical harmonic modes. Since the power in each spherical harmonic mode decreases rapidly with spherical harmonic index, with the $(2,2)$ mode dominating (for an equal-mass nonspinning binary), it is possible to do a very accurate comparison that is valid for all angles by using only a few modes. In addition, as pointed out by Kidder [62], the dominant $(2,2)$ mode can be computed to 3PN order. For an equal-mass binary, the $(2,2)$ mode is

$$
\begin{aligned}
h_{(2,2)}= & -2 \sqrt{\frac{\pi}{5}} \frac{G m}{c^{2} R} e^{-2 i \Phi} x\left\{1-\frac{373}{168} x+\left[2 \pi+6 i \ln \left(\frac{x}{x_{0}}\right)\right] x^{3 / 2}\right. \\
& -\frac{62653}{24192} x^{2}-\left[\frac{197}{42} \pi+\frac{197 i}{14} \ln \left(\frac{x}{x_{0}}\right)+6 i\right] x^{5 / 2} \\
& +\left[\frac{43876092677}{1117670400}+\frac{99}{128} \pi^{2}-\frac{428}{105} \ln x-\frac{856}{105} \gamma\right. \\
& -\frac{1712}{105} \ln 2-18\left[\ln \left(\frac{x}{x_{0}}\right)\right]^{2}+\frac{428}{105} i \pi \\
& \left.\left.+12 i \pi \ln \left(\frac{x}{x_{0}}\right)\right] x^{3}\right\}
\end{aligned}
$$


Since the $(2,2)$ mode of the numerical waveforms is less noisy than the waveform measured along the $z$-axis, and since we have access to the 3PN amplitude correction of the $(2,2)$ mode, we will use the $(2,2)$ waveforms rather than the $z$-axis waveforms for our comparisons between NR and PN in Sec. VI. We have verified (for all comparisons using post-Newtonian waveforms of $\leq 2.5 \mathrm{PN}$ order in amplitude) that our results do not change significantly when we use $z$-axis waveforms instead of $(2,2)$ waveforms.

\section{Absorbing amplitude terms into a redefinition of the phase}

The logarithms of the orbital frequency parameter $x$ (as well as the constant frequency scale $x_{0}$ ) that appear in the amplitude expressions (28), (29), and (32) can be absorbed into a redefinition of the phase by introducing an auxiliary phase variable $\Psi=\Phi+\delta$. Noting that the $\ln x$ terms first enter at $1.5 \mathrm{PN}$ order, it is straightforward to show that choosing $[62,129,133]$

$$
\delta=-3 \frac{M_{\mathrm{ADM}}}{m} x^{3 / 2} \ln \left(\frac{x}{x_{0}}\right),
$$

where $M_{\mathrm{ADM}} / m=1-x / 8+O\left(x^{2}\right)$ for an equal-mass system, will eliminate the $\ln x$ terms from both the $(2,2)$ mode as well as for the polarization waveforms. This follows from

$$
\begin{aligned}
h_{(2,2)} & =A e^{-2 i \Psi}=A e^{-2 i \Phi} e^{-2 i \delta} \\
& =A e^{-2 i \Phi}\left(1-2 i \delta-2 \delta^{2}+O\left(x^{9 / 2}\right)\right),
\end{aligned}
$$

and similarly for the polarization waveforms. Furthermore, since the orbital phase as a function of frequency goes as $x^{-5 / 2}$ at leading order (see Eq. (40) below), the $\ln x$ terms, which were $1.5 \mathrm{PN}, 2.5 \mathrm{PN}$, and $3 \mathrm{PN}$ order in the original amplitude expressions, now appear as phase corrections at relative order 4PN, 5PN, and 5.5PN. As these terms are beyond the order to which the orbital phase evolution is known (3.5PN order), it can be argued that these terms can be ignored. Note that the choices of $x_{0}$ in Eq. (30) and $\delta$ in Eq. (33) are not unique; they were made to gather all logarithmic terms into one term, as well as to simplify the waveform [133].

\section{Energy balance}

The second assumption that goes into making as accurate a post-Newtonian waveform as possible is that of energy balance. It is assumed that the energy carried away by the emission of gravitational waves is balanced by the change in the orbital binding energy of the binary,

$$
\frac{d E}{d t}=-\mathcal{L} \text {. }
$$

While this is extremely plausible, it has only been confirmed through 1.5 PN order [134].
Given the above expressions for the energy, flux, and waveform amplitude, there is still a set of choices that must be made in order to produce a post-Newtonian waveform that can be compared to our numerical waveform. These include

(1) The PN order through which terms in the orbital energy and luminosity are retained.

(2) The procedure by which the energy balance equation is used to obtain $x(t)$ and $\Phi(t)$.

(3) The PN order through which terms in the waveform amplitude are kept.

(4) The treatment of the $\ln x$ terms. These terms can be included in the amplitude or included in the orbital phase via the auxiliary phase $\Psi \equiv \Phi+\delta$. If the latter is chosen, these terms can be retained or ignored; ignoring them can be justified because they occur at higher order than all known terms in the orbital phase.

We always expand energy and luminosity to the same order, which may be different from the order of the amplitude expansion; both of these expansion orders are indicated explicitly in each of our comparisons. We ignore the $\ln \left(x / x_{0}\right)$ terms in the amplitude by absorbing them into the phase and dropping them because of their high PN order. In the next section we describe several choices for obtaining $x(t)$ and $\Phi(t)$ from the energy balance equation.

\section{E. Taylor approximants: Computing $\Phi(t)$}

In this section we describe how to obtain the orbital phase as a function of time, $\Phi(t)$, using the energy balance Eq. (34). Different methods of doing this exist; here we follow the naming convention of [54]. These methods, and variations of them, are called Taylor approximants, and all formally agree to a given PN order but differ in how higherorder terms are truncated. We discuss four time-domain approximants here, but more can be defined.

\section{TaylorT1}

The TaylorT1 approximant is obtained by numerically integrating the ODEs

$$
\begin{gathered}
\frac{d x}{d t}=-\frac{\mathcal{L}}{(d E / d x)}, \\
\frac{d \Phi}{d t}=\frac{c^{3}}{G m} x^{3 / 2},
\end{gathered}
$$

to produce $\Phi(t)$. The fraction on the right side of Eq. (35) is retained as a ratio of post-Newtonian expansions, and is not expanded further before numerical integration. This is the approximant used in the NR-PN comparisons in $[35,41]$.

\section{TaylorT2}

The TaylorT2 approximant is obtained by starting with the parametric solution of the energy balance equation: 


$$
\begin{gathered}
t(x)=t_{0}+\int_{x}^{x_{0}} d x \frac{(d E / d x)}{\mathcal{L}}, \\
\Phi(x)=\Phi_{0}+\int_{x}^{x_{0}} d x \frac{x^{3 / 2} c^{3}}{G m} \frac{(d E / d x)}{\mathcal{L}} .
\end{gathered}
$$

The integrand of each expression is reexpanded as a single post-Newtonian expansion in $x$ and truncated at the appropriate PN-order; these integrals are then evaluated analytically to obtain for an equal-mass binary $[54,55]$ :

$$
\begin{aligned}
t= & t_{0}-\frac{5 G m}{64 c^{3}} x^{-4}\left\{1+\frac{487}{126} x-\frac{32}{5} \pi x^{3 / 2}+\frac{2349439}{254016} x^{2}\right. \\
& -\frac{1864}{63} \pi x^{5 / 2}+\left[-\frac{999777207379}{5867769600}+\frac{1597}{48} \pi^{2}\right. \\
& \left.\left.+\frac{6848}{105} \gamma+\frac{3424}{105} \ln (16 x)\right] x^{3}-\frac{571496}{3969} \pi x^{7 / 2}\right\}
\end{aligned}
$$

$$
\begin{aligned}
\Phi= & \Phi_{0}-\frac{1}{8} x^{-5 / 2}\left\{1+\frac{2435}{504} x-10 \pi x^{3 / 2}\right. \\
& +\frac{11747195}{508032} x^{2}+\frac{1165}{42} \pi x^{5 / 2} \ln x \\
& +\left[\frac{1573812724819}{4694215680}-\frac{7985}{192} \pi^{2}-\frac{1712}{21} \gamma\right. \\
& \left.\left.-\frac{856}{21} \ln (16 x)\right] x^{3}+\frac{357185}{7938} \pi x^{7 / 2}\right\} .
\end{aligned}
$$

\section{TaylorT3}

The TaylorT3 approximant is closely related to TaylorT2. It is obtained by introducing the dimensionless time variable

$$
\tau \equiv \frac{\nu c^{3}}{5 G m}\left(t_{0}-t\right)
$$

where $\nu=m_{1} m_{2} / m^{2}$ and $\tau^{-1 / 4}=O(\epsilon)$. The TaylorT2 expression $t(x)$ is inverted to obtain $x(\tau)$, and truncated at the desired PN order. Then $x(\tau)$ is integrated to obtain

$$
\Phi(\tau)=\Phi_{0}-\int_{\tau_{0}}^{\tau} d \tau \frac{5 x^{3 / 2}}{\nu} .
$$

This procedure yields for an equal-mass binary [104]:

$$
\begin{aligned}
x= & \frac{1}{4} \tau^{-1 / 4}\left\{1+\frac{487}{2016} \tau^{-1 / 4}-\frac{1}{5} \pi \tau^{-3 / 8}\right. \\
& +\frac{1875101}{16257024} \tau^{-1 / 2}-\frac{1391}{6720} \pi \tau^{-5 / 8} \\
& +\left[-\frac{999777207379}{1502149017600}+\frac{1597}{12288} \pi^{2}+\frac{107}{420} \gamma\right. \\
& \left.\left.-\frac{107}{3360} \ln \left(\frac{\tau}{256}\right)\right] \tau^{-3 / 4}-\frac{88451}{282240} \pi \tau^{-7 / 8}\right\},
\end{aligned}
$$

$$
\begin{aligned}
\Phi= & \Phi_{0}-4 \tau^{5 / 8}\left\{1+\frac{2435}{4032} \tau^{-1 / 4}-\frac{3}{4} \pi \tau^{-3 / 8}\right. \\
& +\frac{1760225}{1806336} \tau^{-1 / 2}-\frac{1165}{5376} \pi \tau^{-5 / 8} \ln \tau \\
& +\left[\frac{24523613019127}{3605157642240}-\frac{42997}{40960} \pi^{2}-\frac{107}{56} \gamma\right. \\
& \left.\left.+\frac{107}{448} \ln \left(\frac{\tau}{256}\right)\right] \tau^{-3 / 4}+\frac{28325105}{21676032} \pi \tau^{-7 / 8}\right\}
\end{aligned}
$$

This is the post-Newtonian approximant used in visual comparisons by [32] and in the NR-PN comparisons in [41] at $3 \mathrm{PN}$ order in phase.

\section{TaylorT4}

In addition to simply numerically integrating the fluxenergy equation (37), as is done for TaylorT1, one may instead reexpand the right side of (37) as a single series and truncate at the appropriate PN order before doing the integration. The phase evolution $\Phi(t)$ can thus be obtained by numerically integrating the ODEs

$$
\begin{gathered}
\frac{d x}{d t}=\frac{16 c^{3}}{5 G m} x^{5}\left\{1-\frac{487}{168} x+4 \pi x^{3 / 2}+\frac{274229}{72576} x^{2}\right. \\
-\frac{254}{21} \pi x^{5 / 2}+\left[\frac{178384023737}{3353011200}+\frac{1475}{192} \pi^{2}\right. \\
\left.\left.-\frac{1712}{105} \gamma-\frac{856}{105} \ln (16 x)\right] x^{3}+\frac{3310}{189} \pi x^{7 / 2}\right\} \\
\frac{d \Phi}{d t}=\frac{x^{3 / 2} c^{3}}{G m} .
\end{gathered}
$$

This approximant was not considered in [54], however for consistency with their notation, we call it TaylorT4. TaylorT4 is the primary approximant used in the NR-PN comparisons in $[38,39]$, and one of the several approximants considered in the NR-PN comparisons in [35]. Reference [32] pointed out that TaylorT4 at 3.5PN order in phase is close to TaylorT3 at 3PN order in phase, and therefore should give similar agreement with numerical results.

\section{PN-NR COMPARISON PROCEDURE}

\section{A. What to compare?}

There are many ways to compare numerical relativity and post-Newtonian results. For example, the postNewtonian orbital phase $\Phi(t)$ could be compared with the coordinate phase of the black-hole trajectories. However, this and many other comparisons are difficult to make in a coordinate-independent manner without expending significant effort to understand the relationship between the gauge choices used in post-Newtonian theory and in the NR simulations. Therefore, in order to obtain the most meaningful comparison possible, we attempt to mini- 
mize gauge effects by comparing gravitational waveforms as seen by an observer at infinity. The waveform quantity most easily obtained from the numerical relativity code is the Newman-Penrose quantity $\Psi_{4}$, and we will compare its $(2,2)$ component [cf. Eq. (13)], split into phase $\phi$ and amplitude $A$ according to Eq. (14) and extrapolated to infinite extraction radius.

The post-Newtonian formulae in Sec. III yield the metric perturbation components $h_{+}$and $h_{\times}$, which - for a gravitational wave at infinity - are related to $\Psi_{4}$ by

$$
\Psi_{4}(t)=\frac{\partial^{2}}{\partial t^{2}}\left(h_{+}(t)-i h_{\times}(t)\right) .
$$

We numerically differentiate the post-Newtonian expressions for $h_{+}(t)$ and $h_{\times}(t)$ twice before computing amplitude and phase using Eq. (14). Note that $\phi(t)$ will differ slightly from the phase computed from the metric perturbation directly, as $\tan ^{-1}\left(h_{\times} / h_{+}\right)$, because both the amplitude and phase of the metric perturbation are time dependent. For the same reason, $\phi(t)$ is not precisely equal to twice the orbital phase.

As in Ref. [41], we compare $\Psi_{4}$ rather than $h_{+, \times}$to avoid difficulties arising with fixing the integration constants when integrating the numerically obtained $\Psi_{4}$ (see [40] for more details). Both $\Psi_{4}$ and $h_{+, \times}$contain the same information, so differences between both procedures should be minimal.

\section{B. Matching procedure}

Each of the post-Newtonian waveforms has an arbitrary time offset $t_{0}$ and an arbitrary phase offset $\phi_{0}$. These constants can be thought of as representing the absolute time of merger and the orientation of the binary at merger, and we are free to adjust them in order to match NR and PN waveforms. Following [38,41], we choose these constants by demanding that the PN and NR gravitational-wave phase and gravitational-wave frequency agree at some fiducial frequency $\omega_{m}$. Specifically, we proceed as follows: We start with a NR waveform $\Psi_{4}^{\mathrm{NR}}(t)$ and an unshifted PN waveform $\Psi_{4}^{\mathrm{PN}^{\prime}}(t)$ that has an arbitrary time and phase shift. After selecting the matching frequency $\omega_{m}$, we can find (to essentially unlimited accuracy) the time $t_{c}$ such that the derivative of the PN phase satisfies $\dot{\phi}_{\mathrm{PN}^{\prime}}\left(t_{c}\right)=\omega_{m}$, where $\phi_{\mathrm{PN}^{\prime}}(t)$ is the phase associated with $\Psi_{4}^{\mathrm{PN}^{\prime}}(t)$. Similarly, we find the time $t_{m}$ such that $\dot{\phi}_{\mathrm{NR}}\left(t_{m}\right)=\omega_{m}$. The time $t_{m}$ cannot be found to unlimited accuracy, and the uncertainty in $t_{m}$ is due mainly to residual eccentricity of the NR waveform, as discussed in Sec. VE. Once we have $t_{m}$ and $t_{c}$, we leave the NR waveform untouched, but we construct a new, shifted, PN waveform

$$
\Psi_{4}^{\mathrm{PN}}(t)=\Psi_{4}^{\mathrm{PN}^{\prime}}\left(t+t_{c}-t_{m}\right) e^{i\left(\phi_{\mathrm{PN}^{\prime}}\left(t_{c}\right)-\phi_{\mathrm{NR}}\left(t_{m}\right)\right)} .
$$

The phase of this new PN waveform is therefore

$$
\phi_{\mathrm{PN}}(t)=\phi_{\mathrm{PN}^{\prime}}\left(t+t_{c}-t_{m}\right)-\phi_{\mathrm{PN}^{\prime}}\left(t_{c}\right)+\phi_{\mathrm{NR}}\left(t_{m}\right),
$$

which satisfies $\phi_{\mathrm{PN}}\left(t_{m}\right)=\phi_{\mathrm{NR}}\left(t_{m}\right)$ and $\dot{\phi}_{\mathrm{PN}}\left(t_{m}\right)=\omega_{m}$ as desired. All our comparisons are then made using the new shifted waveform $\Psi_{4}^{\mathrm{PN}}(t)$ rather than the unshifted waveform $\Psi_{4}^{\mathrm{PN}^{\prime}}(t)$.

\section{Choice of masses}

The post-Newtonian expressions as written in Sec. III involve the total mass $m$, which corresponds to the sum of the bare masses of the point particles in post-Newtonian theory. When comparing PN to NR, the question then arises as to which of the many definitions of the mass of a numerically generated binary black-hole solution should correspond to the post-Newtonian parameter $m$. For nonspinning black holes at very large separation, $m$ reduces to the sum of the irreducible masses of the two holes. Neglecting tidal heating, the irreducible masses should be conserved during the inspiral, so that we identify $m$ with the sum of the irreducible masses of the initial data 30c. As discussed in Sec. V the black-hole spins are sufficiently small so that there is no discernible difference between irreducible mass of the black holes and the Christodoulou mass, Eq. (3). Of course, the latter would be more appropriate for spinning black holes.

\section{ESTIMATION OF UNCERTAINTIES}

To make precise statements about agreement or disagreement between numerical and post-Newtonian waveforms, it is essential to know the size of the uncertainties in this comparison. When discussing these uncertainties, we must strive to include all effects that may cause our numerical waveform to differ from the post-Newtonian waveforms we compare to. For instance, in addition to considering effects such as numerical truncation error, we also account for the fact that NR and PN waveforms correspond to slightly different physical scenarios: The PN waveforms have identically zero spin and eccentricity, whereas the numerical simulations have some small residual spin and eccentricity. Table III lists all effects we have considered; we discuss these in detail below starting in Sec. VA. All uncertainties are quoted in terms of phase and amplitude differences, and apply to waveform comparisons via matching at a fixed $\omega_{m}$ according to the procedure in Sec. IV B.

Most of the effects responsible for our uncertainties are time dependent, so that it is difficult to arrive at a single number describing each effect. For simplicity, the error bounds in Table III ignore the junk-radiation noise that occurs in the numerical waveform for $t-r^{*} \leqslant 1000 m$. The extent to which this noise affects the PN-NR comparisons presented below in Secs. VIA and VIB will be evident from the noise in the graphs in these sections. Note that all four matching frequencies $\omega_{m}$ occur after the noise disappears at $t-r^{*} \sim 1000 m$. Furthermore, the post-Newtonian waveforms end at different times depend- 
ing on the PN order and on which particular postNewtonian approximant is used. Therefore, in order to produce a single number for each effect listed in Table III, we consider only the part of the waveform prior to some cutoff time, which we choose to be the time at which the numerical waveform reaches gravitational-wave frequency $m \omega=0.1$.

\section{A. Errors in numerical approximations}

The first three error sources listed in Table III have already been discussed in detail in Sec. II. We estimate numerical truncation error using the difference between the two highest-resolution runs after the waveforms have been shifted to agree at some matching frequency $\omega_{m}$. For $m \omega_{m}=0.1$ this difference is shown as the curves labeled "30c-1/N5" in the lower panels of Figs. 7 and 8, and corresponds to a phase difference of 0.003 radians and a relative amplitude difference of 0.001 . For other values of $\omega_{m}$ the differences are similar. The effect of the outer boundary is estimated by the difference between the runs $30 \mathrm{c}-1 / \mathrm{N} 6$ and $30 \mathrm{c}-2 / \mathrm{N} 6$, which for $m \omega_{m}=0.1$ is shown as the curves labeled " $30 \mathrm{c}-2 / \mathrm{N} 6$ " in the lower panels of Figs. 7 and 8, and amount to phase differences of 0.005 radians and relative amplitude differences of 0.002. Errors associated with extrapolation to infinity have been discussed in detail in Figs. 10 and 12. Specifically, Fig. 10 shows that increasing the extrapolation order between 3 and 4 changes the extrapolated phase by less than 0.005 radians, and Fig. 12 confirms that the extrapolated result is robust under changes of extraction radii.

TABLE III. Summary of uncertainties in the comparison between numerical relativity and post-Newtonian expansions. Quoted error estimates ignore the junk-radiation noise at $t \lesssim$ $1000 \mathrm{~m}$ and apply to times before the numerical waveform reaches gravitational-wave frequency $m \omega=0.1$. Uncertainties apply to waveform comparisons via matching at a fixed $\omega_{m}$ according to the procedure in Sec. IV B, and represent the maximum values for all four different matching frequencies $\omega_{m}$ that we consider, unless noted otherwise.

\begin{tabular}{lcc}
\hline \hline Effect & $\delta \phi$ (radians) & $\delta A / A$ \\
\hline Numerical truncation error & 0.003 & 0.001 \\
Finite outer boundary & 0.005 & 0.002 \\
Extrapolation $r \rightarrow \infty$ & 0.005 & 0.002 \\
Wave extraction at $r_{\text {areal }}=$ const? & 0.002 & $10^{-4}$ \\
Drift of mass $m$ & 0.002 & $10^{-4}$ \\
Coordinate time $=$ proper time? & 0.002 & $10^{-4}$ \\
Lapse spherically symmetric? & 0.01 & $4 \times 10^{-4}$ \\
Residual eccentricity & $0.02^{\mathrm{a}}$ & 0.004 \\
Residual spins & 0.03 & 0.001 \\
Root-mean-square sum & $0.04^{\mathrm{a}}$ & 0.005 \\
\hline \hline
\end{tabular}

${ }^{\mathrm{a}}$ For the case of matching at $m \omega_{m}=0.04$, the phase uncertainty due to residual eccentricity increases to 0.05 radians, thus increasing the root-mean-square sum to 0.06 radians.

\section{B. Constancy of extraction radii}

If the physical locations of the coordinate-stationary extraction radii happen to change during the evolution, then the extracted gravitational waves will accrue a timing error equal to the light-travel time between the original location and the final location. From Fig. 9, we see that the drift in areal radius is less than $0.02 m$, resulting in a time uncertainty of $\delta t=0.02 \mathrm{~m}$. This time uncertainty translates into a phase uncertainty via

$$
\delta \phi=m \omega \times(\delta t / m)
$$

which yields $\delta \phi \approx 0.002$, when $m \omega=0.1$ (the value at the end of the PN comparison) was used.

To estimate the effect of this time uncertainty on the amplitude, we first note that to lowest order in the postNewtonian parameter $x$ (defined in Eq. (23)), the wave amplitude of $\Psi_{4}$ scales like $x^{4}$. Also, from Eq. (45), we have $d x / d t=16 /(5 m) x^{5}$. Therefore,

$$
\frac{\delta A}{A} \sim \frac{d \ln A}{d x} \frac{d x}{d t} \delta t \sim \frac{64}{5}(m \omega / 2)^{8 / 3} \frac{\delta t}{m},
$$

where we have used the fact that the gravitational-wave frequency $\omega$ is approximately twice the orbital frequency. For a time uncertainty $\delta t=0.02 m$, Eq. (51) gives $\delta A / A \approx$ $10^{-4}$ for $m \omega=0.1$.

\section{Constancy of mass}

Our comparisons with post-Newtonian formulae assume a constant post-Newtonian mass parameter $m$, which we set equal to the total irreducible mass of the black holes in the numerical simulation. If the total mass of the numerical simulation is not constant, this will lead to errors in the comparison. For example, changes in $t / m$ caused by a changing mass will lead to phase differences. Figure 4 demonstrates that the irreducible mass is conserved to a fractional accuracy of about $\delta m / m \approx 5 \times 10^{-6}$.

This change in irreducible mass could be caused by numerical errors, or by a physical increase of the mass of each black hole through tidal heating. For our simulations, $m(t)$ decreases during the run (this is not apparent from Fig. 4 which plots absolute values), thus contradicting the second law of black-hole thermodynamics. Moreover, the increase in $m(t)$ through tidal heating is much smaller than the observed variations in $m(t)$ (see, e.g. [135]). Therefore, the variations in $m(t)$ are numerical errors, and we need to bound the influence of these errors on the comparison to post-Newtonian expansions.

Over an evolution time of $t / m=4000$, the observed mass uncertainty of $\delta m / m \approx 5 \times 10^{-6}$ results in an uncertainty in the overall time interval of $\delta(t / m)=(t / m) \times$ $(\delta m / m) \approx 0.02$. This time uncertainty translates into a phase uncertainty of $\delta \phi \approx 0.002$, using Eq. (50) for $m \omega=0.1$. Note that the effect of the black-hole spins on the mass is negligible relative to the numerical drift of $5 \times$ $10^{-6}$. This is because the spins of the holes are bounded by 
$S / M_{\text {irr }}<2 \times 10^{-4}$ and the spin enters quadratically into the Christodoulou formula (3). The error in the gravitational-wave amplitude caused by time uncertainties due to varying mass is $\delta A / A \approx 10^{-4}$ using Eq. (51) for $m \omega=0.1$. An error in the mass will affect the amplitude not only via a time offset, but also because the amplitude is proportional to $(\omega m / 2)^{8 / 3}$ (to lowest PN order). However, this additional error is very small, $\delta A / A \approx(8 / 3) \delta m / m \approx$ $10^{-5}$.

\section{Time coordinate ambiguity}

We now turn to two possible sources of error that have not yet been discussed, both of which are related to ambiguity in the time coordinate. The basic issue is that the time variable $t$ in post-Newtonian expansions corresponds to proper time in the asymptotically flat region, but the time $t$ in numerical simulations is coordinate time. These two quantities agree only if the lapse function $N$ approaches unity at large distances. To verify this, we decompose $N$ in spherical harmonics centered on the center of mass of the system,

$$
N(r, \theta, \varphi)=\sum_{l=0}^{\infty} \sum_{m=-l}^{l} N_{l m}(r) Y_{l m}(\theta, \varphi) .
$$

The angular average of the lapse function, $\bar{N}(r) \equiv$ $\sqrt{4 \pi} N_{00}(r)$ should then approach unity for $r \rightarrow \infty$, and all other modes $N_{l m}(r)$ should decay to zero. The top panel of Fig. 14 plots $\bar{N}(r)-1$ vs $m / r$ for three different evolution times. Fitting $\bar{N}(r)-1$ for $r>100 m$ to a polynomial in $m / r$ gives a $y$-intercept of $<5 \times 10^{-6}$ for all three times, and for polynomial orders of two through five. Therefore, the coordinate time of the evolution agrees with proper time at infinity to better than $\delta t / m=t / m \times 5 \times 10^{-6} \approx$ 0.02 , which induces a phase error of at most $\delta \phi \approx 0.002$ and an amplitude error of $\delta A / A \approx 10^{-4}$ [cf. Eqs. (50) and (51)].

The second source of error related to the lapse is shown in the lower panel of Fig. 14, which presents the three dominant higher-order moments $N_{l m}(r)$. All these modes decay to zero as $r \rightarrow \infty$, except, perhaps, the real part of the $N_{22}$ mode at $t / m=3800$. This mode seems to approach a value of about $5 \times 10^{-5}$. At $t=1900 \mathrm{~m}$, this mode still decays nicely to zero, hence the maximum time uncertainty introduced by this effect at late times is $\delta t=1900 \mathrm{~m} \times 5 \times 10^{-5} \approx 0.1 \mathrm{~m}$, resulting in a potential phase uncertainty of $\delta \phi \approx 0.01$ and a potential amplitude uncertainty of $\delta A / A \approx 4 \times 10^{-4}$.

\section{E. Eccentricity}

We estimated the eccentricity during the numerical simulation with several of the methods described in $[32,40,61]$, and have found consistently $e \leqq 6 \times 10^{-5}$. This eccentricity can affect our comparison to a post-

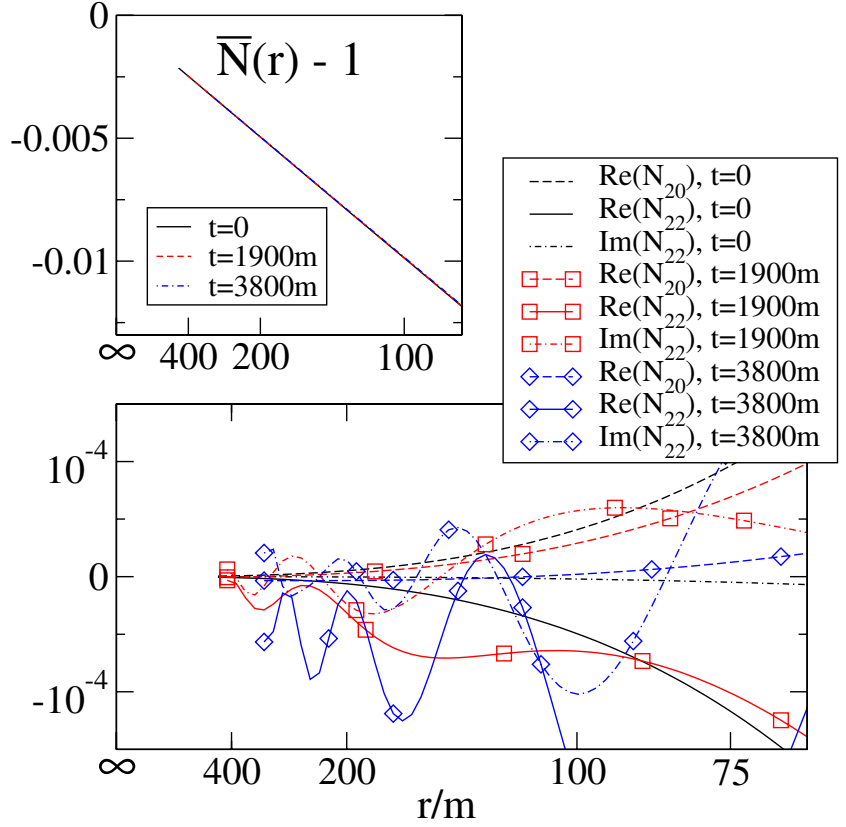

FIG. 14 (color online). Asymptotic behavior of the lapse at large radii for times $t / m=0,1900,3800$. The top figure displays the angular average of the lapse as a function of radius at $t=0,1900 m, 7800 m$. The bottom figure shows the dominant higher multipole moments of the lapse. Both horizontal axes are spaced in $1 / r$.

Newtonian waveform of a quasicircular (i.e. zeroeccentricity) inspiral in three ways.

\section{Change in rate of inspiral}

The first effect arises because an eccentric binary has a different inspiral rate than a noneccentric binary; physically, this can be understood by noting that the gravitational flux and orbital energy depend upon the eccentricity, and therefore modify the rate at which the orbital frequency evolves assuming energy balance. Reference [136] has derived the first-order correction in the phase of the gravitational wave due to this effect. Converting their result to our notation and restricting to the equal-mass case yields

$$
\frac{1}{(d x / d t)}=\frac{5 G m}{16 c^{3} x^{5}}\left(1-\frac{157}{24} e_{i}^{2}\left(\frac{x_{i}}{x}\right)^{19 / 6}\right),
$$

where $e_{i}$ is the initial eccentricity and $x_{i}$ is the initial value of the orbital frequency parameter. Substituting this into Eq. (38) yields

$$
\Phi=\Phi_{0}-\frac{1}{8} x^{-5 / 2}+\frac{785}{2176} e_{i}^{2} x_{i}^{19 / 6} x^{-17 / 3} .
$$

Using $e_{i}=6 \times 10^{-5}$ and integrating over the frequency range from the start of our simulation to the matching frequency of $m \omega=0.1$ yields a phase shift of $\sim-2 \times$ $10^{-6}$, which is dwarfed by many other error sources, such as the uncertainty in the numerical mass $m$, cf. Sec. VC. 


\section{Uncertainty in matching time}

The second way in which eccentricity affects our comparison is by introducing errors in our procedure for matching the PN and NR waveforms. Recall that our matching procedure involves determining the time $t_{m}$ at which the gravitational-wave frequency $\omega$ takes a certain value $m \omega_{m}$; eccentricity modulates the instantaneous gravitationalwave frequency $\omega(t)$ via

$$
\omega(t)=\bar{\omega}(t)\left[1+2 e \cos \left(\Omega_{r} t\right)\right],
$$

where $\bar{\omega}(t)$ represents the averaged "noneccentric" evolution of the gravitational-wave frequency, and $\Omega_{r}$ is the frequency of radial oscillations, which is approximately equal to the orbital frequency. We see that $\omega$ can differ from $\bar{\omega}$ by as much as $2 e \bar{\omega} \approx 2 e \omega$. This could induce an error in the determination of $t_{m}$ by as much as

$$
\left|\delta t_{m}\right|=\frac{|\delta \omega|}{\dot{\omega}} \approx \frac{2 e \omega}{\dot{\omega}} .
$$

We can simplify this expression by using Eq. (45) to lowest order, and by noting that the gravitational-wave frequency is approximately twice the orbital frequency. We find

$$
\left|\delta t_{m}\right| \leq e \frac{5 m}{12}\left(\frac{m \omega}{2}\right)^{-8 / 3} .
$$

This uncertainty is largest at small frequencies, because the frequency changes much more slowly. For $m \omega=0.04$, we find $\left|\delta t_{m}\right| \lesssim 0.9 m$, and for $m \omega=0.1$, we find $\left|\delta t_{m}\right| \lesssim$ $0.1 \mathrm{~m}$.

To determine how uncertainties in $t_{m}$ translate into phase differences, recall that in the matching procedure described in Sec. IV B, $t_{m}$ enters into the phase of the shifted PN waveform according to Eq. (49). Therefore the phase difference that we compute between the PN and NR waveforms is

$$
\begin{aligned}
\Delta \phi(t)= & \phi_{\mathrm{PN}}(t)-\phi_{\mathrm{NR}}(t) \\
= & \phi_{\mathrm{PN}^{\prime}}\left(t+t_{c}-t_{m}\right)-\phi_{\mathrm{NR}}(t)+\phi_{\mathrm{NR}}\left(t_{m}\right) \\
& -\phi_{\mathrm{PN}^{\prime}}\left(t_{c}\right) .
\end{aligned}
$$

Then the error in $\Delta \phi$ is found by Taylor expanding Eq. (58):

$$
\begin{aligned}
\delta \phi & \equiv \delta(\Delta \phi(t))=\left(\dot{\phi}_{\mathrm{PN}^{\prime}}\left(t+t_{c}-t_{m}\right)-\dot{\phi}_{\mathrm{NR}}\left(t_{m}\right)\right) \delta t_{m} \\
& =\left(\dot{\phi}_{\mathrm{PN}}(t)-\omega_{m}\right) \delta t_{m} .
\end{aligned}
$$

Our simulations (and therefore the comparisons to postNewtonian theory) start at $m \omega \approx 0.033$, so that the maximal error $\delta \phi$ within our comparison at times before the matching frequency will be

$$
\left|\delta \phi_{\text {before }}\right| \leq\left|0.033-\omega_{m}\right|\left|\delta t_{m}\right| .
$$

Combining Eqs. (56) and (60), and using $e \approx 6 \times 10^{-5}$, we find that $\delta \phi_{\text {before }}<0.01$ radians for all four of our matching frequencies $m \omega_{m}=0.04,0.05,0.063,0.1$. The maximum error $\delta \phi$ within our comparison at times after the matching frequency is

$$
\left|\delta \phi_{\text {after }}\right| \leq\left|0.1-\omega_{m}\right|\left|\delta t_{m}\right|,
$$

because we end our comparisons to post-Newtonian theory at $m \omega=0.1$. Equation (61) evaluates to 0.05 radians for $m \omega_{m}=0.04$, and is less than about 0.02 radians for the three higher matching frequencies.

The error in the gravitational-wave amplitude caused by an error in $t_{m}$ can be estimated by Eq. (51). A conservative estimate using $\delta t=0.9 m$ still gives a small error, $\delta A / A \approx$ 0.004 .

Note that the bounds on $\delta \phi_{\text {before }}$ and $\delta \phi_{\text {after }}$ are proportional to the eccentricity of the numerical simulation. Even with eccentricity as low as $6 \times 10^{-5}$, this effect is one of our largest sources or error for the PN-NR comparison. (cf. Table III). This is the reason why the simpler eccentricity removal procedure of Husa et al. [61] (resulting in $e=0.0016$ ) is not adequate for our purposes.

\section{Periodic modulation of phase and amplitude}

The third effect of orbital eccentricity is a periodic modulation of the gravitational-wave phase and amplitude. If we assume that $\bar{\omega}(t)$ varies on much longer time scales than $1 / \Omega_{r}$ (which is true at large separation) then time integration of Eq. (55) yields

$$
\phi(t)=\bar{\phi}(t)+2 e \frac{\bar{\omega}}{\Omega_{r}} \sin \left(\Omega_{r} t\right) .
$$

Because $\Omega_{r} \approx \Omega \approx \bar{\omega} / 2$, we therefore find that the gravitational-wave phase consists of the sum of the desired "circular" phase, $\bar{\phi}(t)$, plus an oscillatory component with amplitude $4 e \approx 2 \times 10^{-4}$. This oscillatory component, however, is much smaller than other uncertainties of the comparison, for instance the uncertainty in determination of $t_{m}$.

Residual eccentricity will also cause a modulation of the gravitational-wave amplitude in a manner similar to that of the phase. This is because eccentricity explicitly enters the post-Newtonian amplitude formula at 0PN order [137]. This term is proportional to $e$, and since $e \leqq 6 \times 10^{-5}$ its contribution to the amplitude error is small compared to the effect due to uncertainty in $t_{m}$.

While oscillations in phase and amplitude due to eccentricity are tiny and dwarfed by other uncertainties in the PN-NR comparison, their characteristic oscillatory behavior makes them nevertheless visible on some of the graphs we present below, for instance, both panels of Fig. 19.

\section{F. Spin}

We now turn our attention to effects of the small residual spins of the black holes. References [138,139] compute spin-orbit coupling up to 2.5 post-Newtonian order, and find that the orbital phase, Eq. (40), acquires the following spin contributions 


$$
\begin{aligned}
\Phi_{S}(x)= & -\frac{1}{32 \nu} \sum_{i=1,2} \chi_{i}\left\{\left(\frac{565}{24} \frac{m_{i}^{2}}{m^{2}}+\frac{125 \nu}{8}\right) x^{-1}\right. \\
& -\left[\left(\frac{681145}{4032}+\frac{965 \nu}{28}\right) \frac{m_{i}^{2}}{m^{2}}+\frac{37265 \nu}{448}\right. \\
& \left.\left.+\frac{1735 \nu^{2}}{56}\right] \ln x\right\}
\end{aligned}
$$

where $\chi_{i}=\mathbf{S}_{i} \cdot \hat{\mathbf{L}} / m_{i}^{2}$ is the projection of the dimensionless spin of the $i$ th hole onto the orbital angular momentum. For equal-mass binaries with spins $\chi_{1}=\chi_{2} \equiv \chi$, this reduces to

$$
\Phi_{S}(x)=-\chi\left(\frac{235}{96} x^{-1}-\frac{270625}{16128} \ln x\right) .
$$

Our comparisons to post-Newtonian theory are performed over the orbital frequency range of $0.0167 \leq m \Omega \leq 0.05$, corresponding to $0.065 \leq x \leq 0.136$. The gravitationalwave phase is approximately twice the orbital phase, so that the spin-orbit coupling contributes

$$
\delta \phi_{S}=2\left[\Phi_{S}(0.065)-\Phi_{S}(0.136)\right] \approx-64 \chi
$$

to the gravitational-wave phase. In Sec. II B we estimated $|\mathbf{S}| / M_{\text {irr }}^{2}<5 \times 10^{-4}$, where $M_{\text {irr }}$ is the irreducible mass of either black hole. Because $\chi \leq|\mathbf{S}| / M_{\text {irr }}^{2} \approx 5 \times 10^{-4}$, the residual black-hole spins contribute less than 0.03 radians to the overall gravitational-wave phase.

We now turn to errors in the amplitude comparison caused by residual spin. From Eq. (64) we can compute the error in orbital frequency as

$$
\begin{aligned}
\delta \Omega & =\dot{\Phi}_{s}=\chi \frac{\dot{x}}{x}\left(\frac{235}{96} x^{-1}+\frac{270625}{16128}\right) \\
& =\chi x^{4} \frac{16}{5 m}\left(\frac{235}{96} x^{-1}+\frac{270625}{16128}\right),
\end{aligned}
$$

where we have used Eq. (47). Because the amplitude of $\Psi_{4}$ scales like $\Omega^{8 / 3}$, we arrive at

$$
\frac{\delta A}{A}=\frac{8}{3} \frac{\delta \Omega}{\Omega}=\chi x^{5 / 2} \frac{128}{15}\left(\frac{235}{96} x^{-1}+\frac{270625}{16128}\right),
$$

which for $m \omega_{m}=0.1$ (i.e. $x=0.136$ ) gives $\delta A / A=$ $2.0 \chi \sim 1.0 \times 10^{-3}$.

Spin-orbit terms also contribute directly to the amplitude $[140,141]$. The leading-order contribution (for an equalmass binary with equal spins) contributes a term $\delta A / A \sim$ $(4 / 3) \chi x^{3 / 2}$, which is the same order of magnitude as the previous error, $10^{-3}$.

\section{RESULTS}

\section{A. Comparison with individual post-Newtonian approximants}

We compare our simulations with four different postNewtonian approximants: the TaylorT1, TaylorT2,
TaylorT3, and TaylorT4 waveforms. These four waveforms agree with each other up to their respective postNewtonian expansion orders, but they differ in the way that the uncontrolled higher-order terms enter. We start with the comparison to TaylorT1.

\section{TaylorT1 (3.5PN phase, 2.5PN amplitude)}

Figure 15 compares the numerical simulation to TaylorT1 3.5/2.5 waveforms (i.e. expansion order 3.5PN in phase and 2.5PN in amplitude, the highest expansion orders currently available for generic direction, cf. III B). The left panel shows the phase difference, where we find differences of more than a radian for all four matching frequencies we consider: $\omega_{m}=0.04,0.05,0.063$, and 0.01 .

For our largest matching frequency, $m \omega_{m}=0.1$, the phase differences are small toward the end of the run by construction. Nevertheless, a phase difference of more than 0.5 radians builds up in the $\sim 1.5$ cycles after the matching point before the TaylorT1 template generation fails. Recall that $m \omega_{m}=0.1$ occurs about 2.2 gravitational-wave cycles before our simulations fail, which is still about 1.5 cycles before merger. However, the largest phase disagreement for $m \omega_{m}=0.1$ builds up at early times, reaching 1.5 radians at the beginning of our simulation, about 28 cycles before the matching $(\sim 30$ cycles before the end of the simulation), and still showing no sign of flattening even at the start of our simulation.

To achieve phase coherence with the early inspiral waveform, it is therefore necessary to match earlier than $m \omega_{m}=0.1$. The left panel of Fig. 15 clearly shows that phase differences at earlier times become smaller when the matching point itself is moved to earlier time. For instance, $m \omega_{m}=0.063$ (about eight gravitational-wave cycles before the end of our simulation), results in phase differences less than 0.5 radians during the 22 earlier cycles of our evolution. However, the phase difference $\phi_{\mathrm{PN}}-\phi_{\mathrm{NR}}$ does not level off at early times within the length of our simulation, so it seems quite possible that the phase difference may grow to a full radian or more if the numerical simulations could cover many more cycles. We thus estimate that for TaylorT1, to achieve 1-radian phase coherence with the early inspiral may require matching more than 10 cycles before merger. To achieve more stringent error bounds in phase coherence will require matching even earlier: for instance it appears one needs to use $m \omega_{m}=$ 0.04 (about 20 cycles before the end of our simulation) for a phase error of less than $\lesssim 0.1$ radians.

While matching at small $\omega_{m}$ yields good phase coherence early in the run, it produces much larger phase differences late in the run. For example, matching at $m \omega_{m}=0.04$ results in a phase difference of almost 2 radians at frequency $m \omega=0.1$. This rather dramatic disagreement is illustrated in Fig. 16, which plots both the numerical and the TaylorT1 waveform, matched at $m \omega_{m}=$ 0.04 . 
GW-cycles before $\mathrm{m} \omega=0.1$

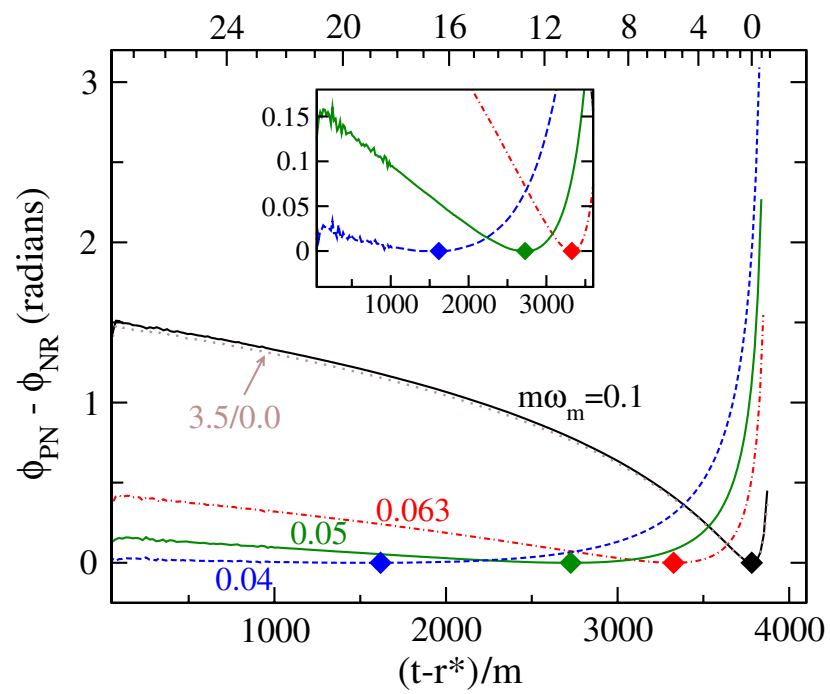

GW-cycles before $m \omega=0.1$

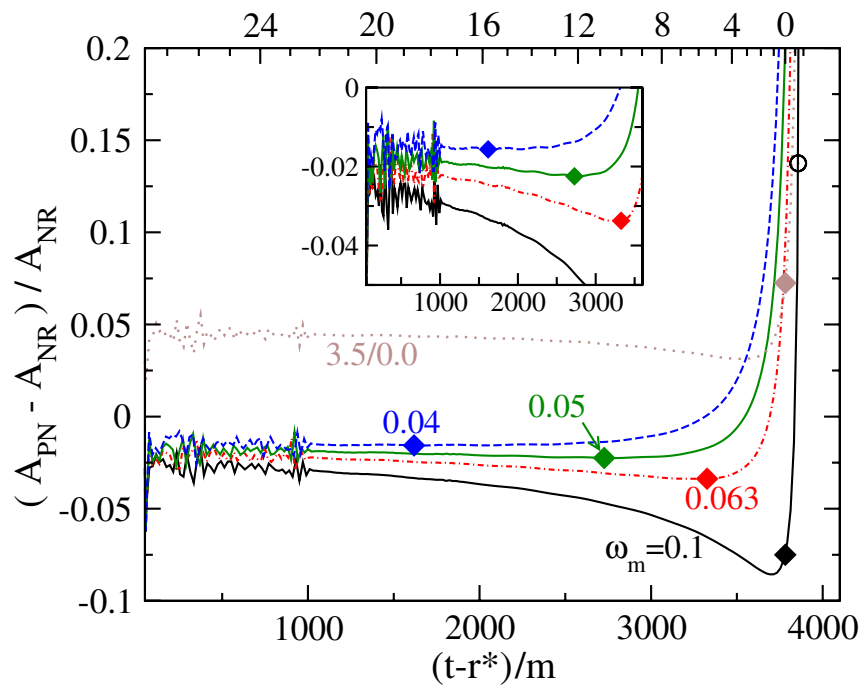

FIG. 15 (color online). Comparison of numerical simulation with TaylorT1 3.5/2.5 waveforms. Left: Difference in gravitationalwave phase. Right: Relative amplitude difference. Plotted are comparisons for four values of $\omega_{m}$. The filled diamond on each curve shows the point at which $\dot{\phi}=\omega_{m}$. The insets show enlargements for small differences and early times. Also shown is the difference between the numerical and restricted (i.e. 3.5PN phase, 0PN amplitude) Taylor T1 for $m \omega_{m}=0.1$.

The left panel of Fig. 15 also includes a comparison to the so-called restricted TaylorT1 template, where only the leading-order amplitude terms are used (i.e. OPN in amplitude). The reason that higher-order amplitude terms affect the phase differences at all is because we are plotting

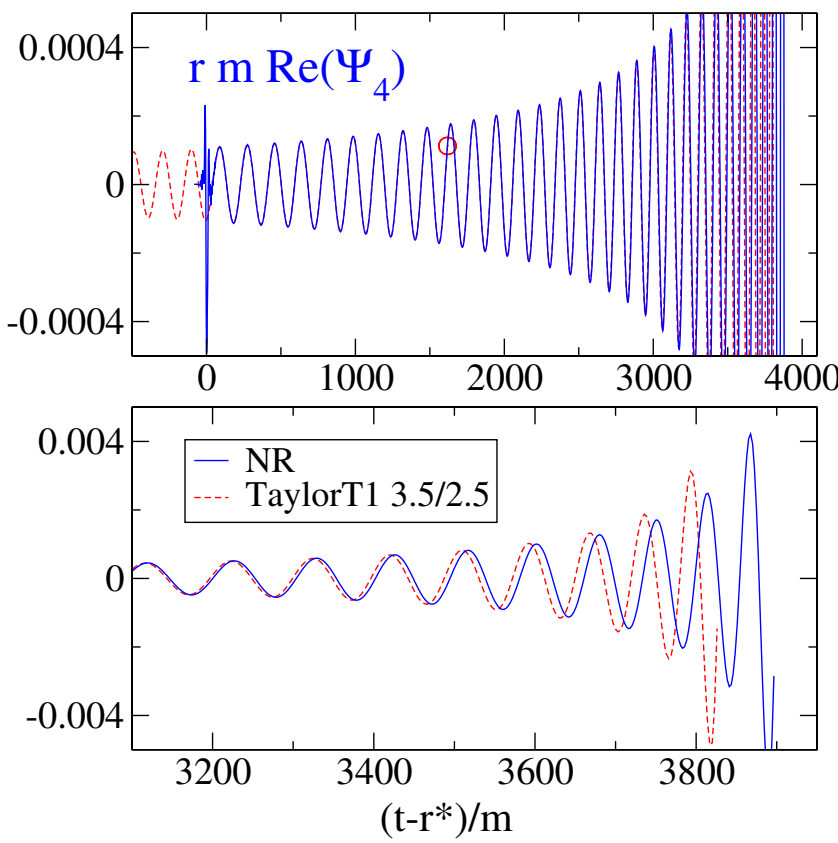

FIG. 16 (color online). Numerical and TaylorT1 3.5/2.5 waveforms. The PN waveform is matched to the numerical one at $m \omega_{m}=0.04$, indicated by the small circle. The lower panel shows a detailed view of the last 10 gravitational-wave cycles. gravitational-wave phase, not orbital phase. However, we see that the effect of these higher-order amplitude terms on the phase difference is small.

We now turn our attention to comparing the amplitudes of the post-Newtonian and numerical waveforms. The right panel of Fig. 15 shows relative amplitude differences between TaylorT1 3.5/2.5 and the numerical waveforms. At early times, the amplitudes agree to within 2 or 3 percent, the agreement being somewhat better when the matching is performed at early times. At late times, the amplitudes disagree dramatically; a large fraction of this disagreement lies probably in the fact the post-Newtonian point of merger (i.e. the point at which the amplitude diverges) occurs at a different time than the numerical point of merger. We also plot the amplitude of the restricted TaylorT1 template. The disagreement between restricted TaylorT1 and the numerical result is much larger, about 5 percent.

Hannam et al. [41] performed a similar comparison, matching their waveforms with a restricted TaylorT1 waveform (i.e. 3.5/0.0) generated using the LIGO Algorithm Library (LAL) [142]. The phase difference they observe for waveforms matched at $m \omega=0.1$ is consistent with our results within numerical errors. When matching TaylorT1 3.5/0.0 early in their simulation (at $m \omega=0.0455)$, however, Hannam et al. find a cumulative phase difference of 0.6 radians at $m \omega=0.1$. From Fig. 15 we find a quite different value of 1.5 radians for our simulation. This disagreement might be caused by the use of the finite extraction radius $R=90 \mathrm{~m}$ for the gravitational-wave phase in Hannam et al.: Fig. 10 shows that extracting at a finite radius leads to a systematic phase 

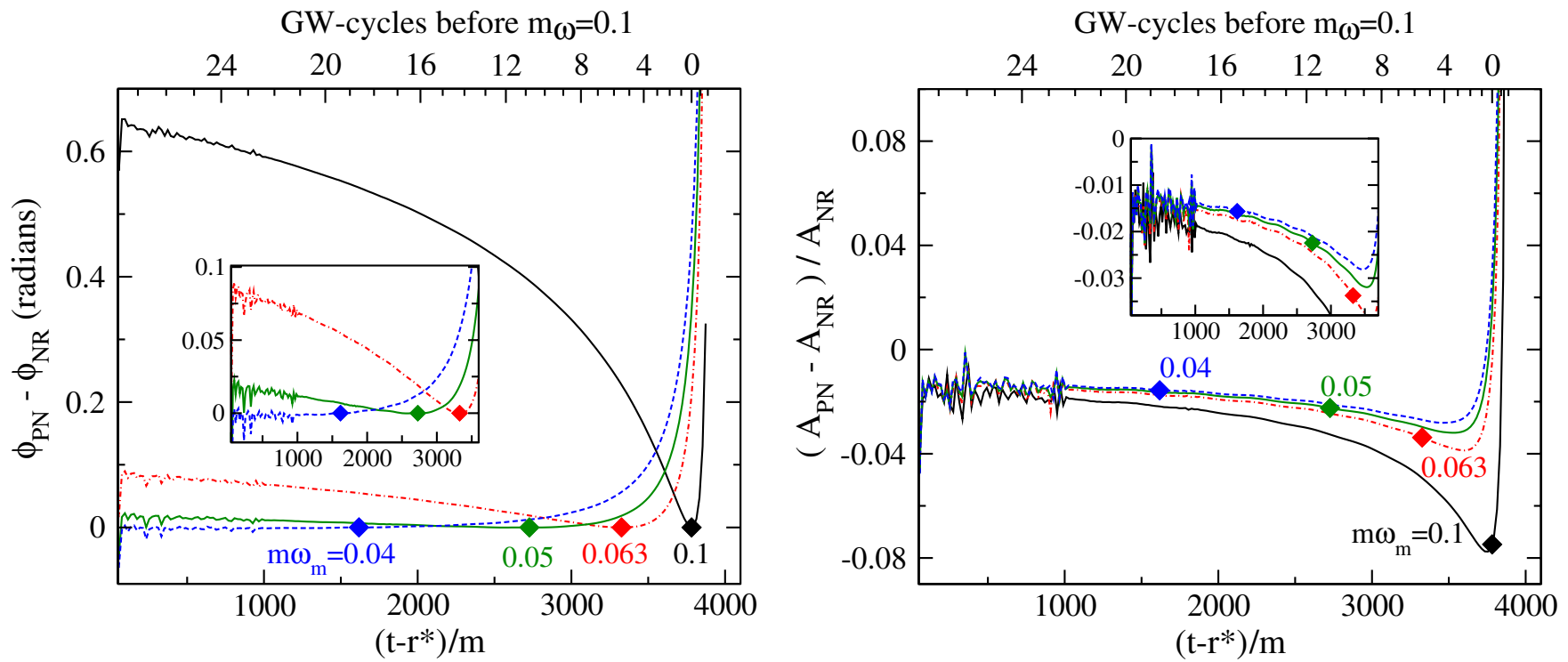

FIG. 17 (color online). Comparison of numerical simulation with TaylorT2 3.5/2.5 waveforms. Left: Difference in gravitationalwave phase. Right: Relative amplitude difference. Plotted are comparisons for four values of $\omega_{m}$. The filled diamond on each curve shows the point at which $\dot{\phi}=\omega_{m}$. The insets show enlargements for small differences and early times.

error, which will induce a systematic error in determination of the matching time of Hannam et al. This error is amplified by the increasing gravitational-wave frequency toward merger.

\section{TaylorT2 (3.5PN phase, 2.5PN amplitude)}

Figure 17 presents the comparison between the numerical waveform and the TaylorT2 approximant. The overall trends are very similar to the TaylorT1 comparison of Fig. 15, however, the phase differences are smaller by about a factor of 2 when matching at $m \omega_{m}=0.1$, and smaller by a factor of 3 to 4 when matching earlier. To our knowledge TaylorT2 has never been compared to a numerical simulation; we include it here mainly for completeness.

\section{TaylorT3 (3.5PN and 3.0PN phase, 2.5PN amplitude)}

Figure 18 is the same as Fig. 15 except it compares numerical simulations to the TaylorT3 family of waveforms. Two differences between TaylorT1 and TaylorT3 are readily apparent from comparing these two figures. The first is that we do not match TaylorT3 3.5/2.5 waveforms at $m \omega_{m}=0.1$. This is because the frequency of TaylorT3 $3.5 / 2.5$ waveforms reaches a maximum shortly before the formal coalescence time of the post-Newtonian template, and then decreases. The maximal frequency is less than 0.1 , so that matching at $m \omega_{m}=0.1$ is not possible. For this reason, we have also shown in Fig. 18 a comparison with a TaylorT3 3.0/3.0 waveform matched at $m \omega_{m}=0.1$. The other major difference between the TaylorT3 3.5/2.5 and TaylorT1 3.5/2.5 comparison is that the phase difference, $\phi_{\mathrm{PN}}-\phi_{\mathrm{NR}}$, has a different sign. While TaylorT1 3.5/2.5 spirals in more rapidly than the numerical simulation, TaylorT3 3.5/2.5 lags behind. Interestingly, the phase differences from the numerical simulation for both TaylorT1 3.5/2.5 and TaylorT3 3.5/2.5 are of about equal magnitude (but opposite sign). The TaylorT3 3.0/3.0 comparison matched at $m \omega_{m}=0.1$ has smaller phase differences than does the TaylorT3 3.5/2.5 comparison, but the slope of the 3.0/3.0 curve in Fig. 18 is nonzero at early times, so it appears that Taylor T3 3.0/3.0 will accumulate significant phase differences at even earlier times, prior to the start of our simulation. In Fig. 22 it can be seen that matching TaylorT3 3.0/3.0 at $m \omega_{m}=0.04$ leads to a good match early, but leads to a phase difference of 0.6 radians by $m \omega=0.1$.

Hannam et al. [41] match a TaylorT3 3.0/0.0 waveform at $m \omega_{m}=0.1$ and $m \omega_{m}=0.0455$. Matching at $m \omega_{m}=$ 0.1 again gives phase differences consistent with our results within numerical errors. Matching at $m \omega_{m}=0.0455$, Hannam et al. find a phase difference of 0.9 radians, while we find a smaller value of 0.5 radians. Again, this difference could be due to the finite extraction radius used by Hannam et al. .

\section{TaylorT4 (3.5PN phase, 2.5PN amplitude)}

Figure 19 is the same as Figs. 15 and 18 except it compares numerical simulations to the TaylorT4 PN waveforms. The agreement between TaylorT4 waveforms and the numerical results is astonishingly good, far better than the agreement between NR and either TaylorT1 or TaylorT3. The gravitational-wave phase difference lies within our error bounds for the entire comparison region $m \omega \leq 0.1$, agreeing to 0.05 radians or better over 29 of 30 gravitational-wave cycles. Reference [38] found agree- 
GW-cycles before $m \omega=0.1$
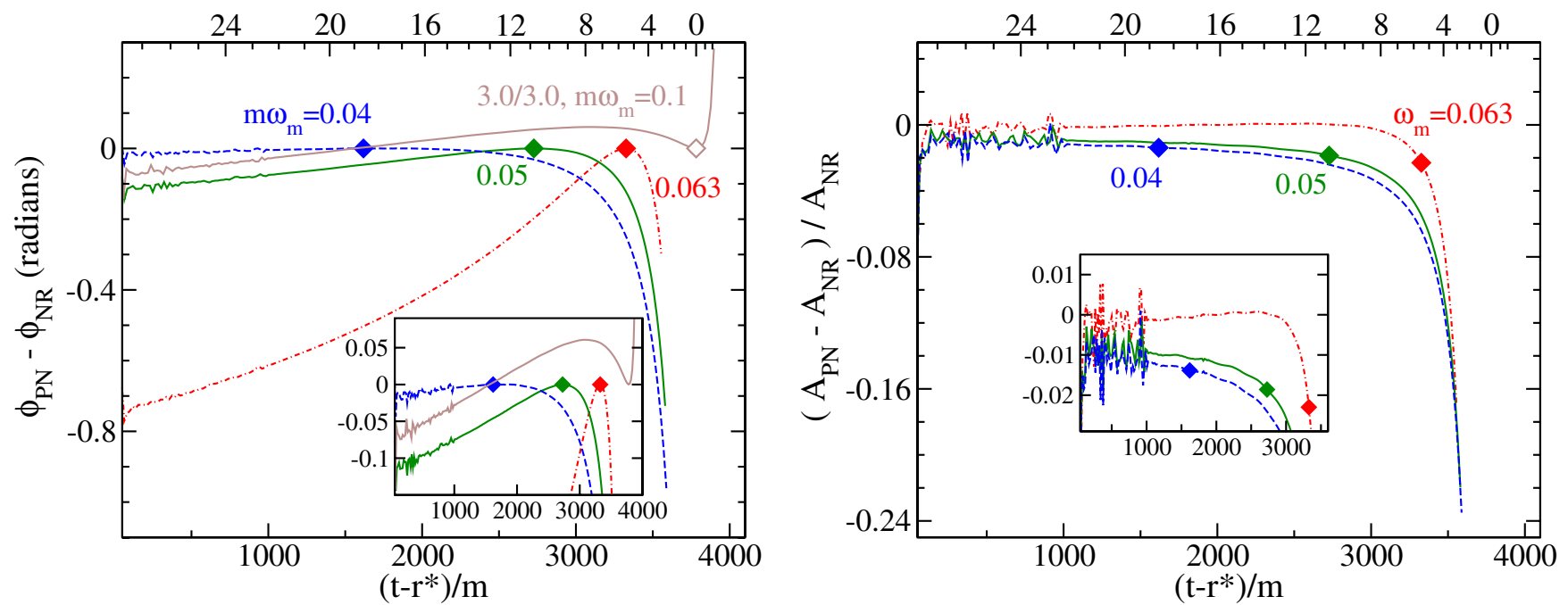

FIG. 18 (color online). Comparison of numerical simulation with TaylorT3 3.5/2.5 waveforms. Left: Difference in gravitationalwave phase. Right: Relative amplitude difference. Plotted are comparisons for three values $\omega_{m}$. The filled diamond on each curve shows the point at which $\dot{\phi}=\omega_{m}$. The lines end when the frequency of the TaylorT3 waveform reaches its maximum, which happens before $m \omega=0.1$, so that the matching frequency $m \omega_{m}=0.1$ is absent. The left plot also contains TaylorT3 3.0/3.0, matched at $m \omega_{m}=0.1$. The insets show enlargements for small differences.

ment between TaylorT4 and their numerical simulation to the level of their numerical accuracy ( $\sim 2$ radians), agreeing to roughly 0.5 radians in the gravitational frequency range of $0.054 \leq m \omega \leq 0.1$. Reference [35] found that NR agrees better with TaylorT4 than with TaylorT1, but the larger systematic and numerical errors of the numerical waveforms used in these studies did not allow them to see the surprising degree to which NR and TaylorT4 agree. The gravitational-wave amplitude of TaylorT4 agrees with the NR waveform to about $1 \%-2 \%$ at early times, and $8 \%$ at late times. In Fig. 20 we plot the NR and TaylorT4 waveforms; the two waveforms are visually indistinguishable on the plot, except for small amplitude differences in the final cycles.

On the left panel of Fig. 19 we also show phase comparisons using $\mathrm{PN}$ waveforms computed to 3.5PN order in
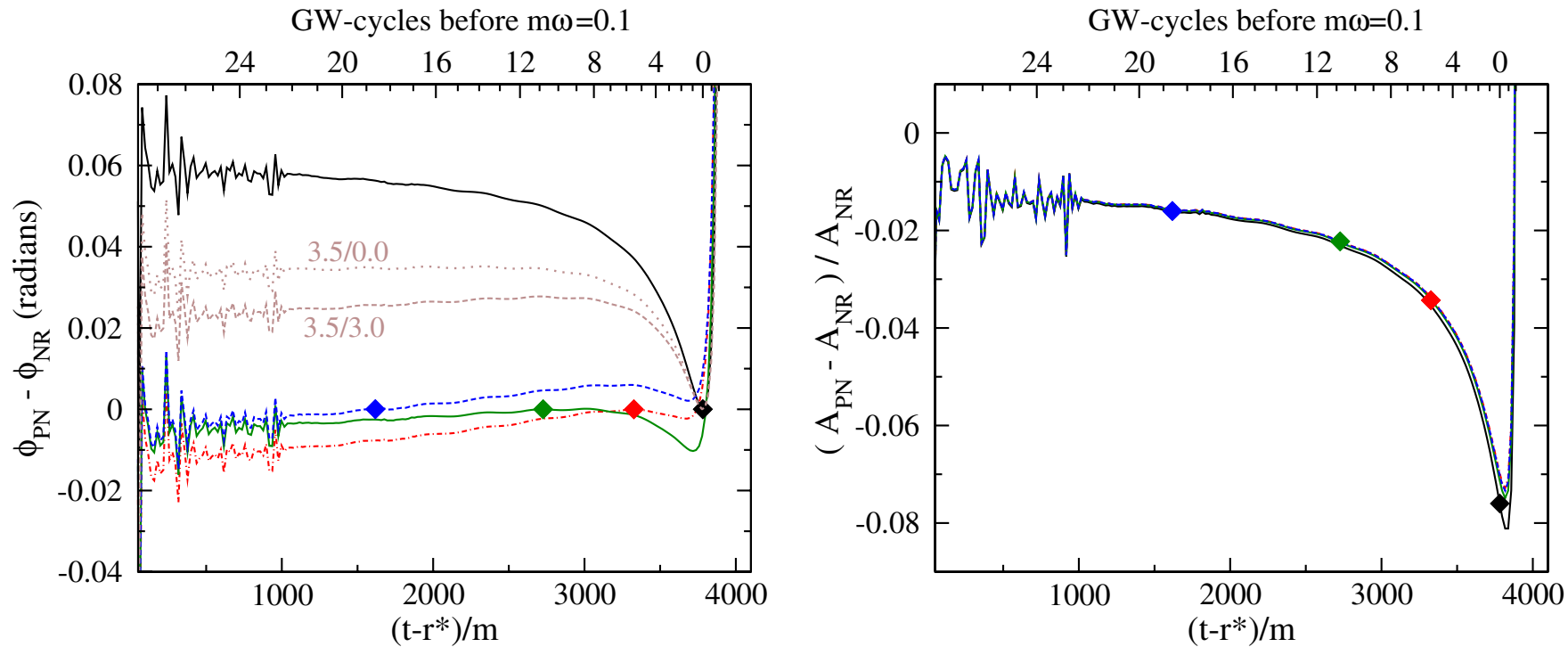

FIG. 19 (color online). Comparison of numerical simulation with TaylorT4 3.5/2.5 waveforms. Left: Difference in gravitationalwave phase. Right: Relative amplitude difference. Plotted are comparisons for four values of $\omega_{m}$. The filled diamond on each curve shows the point at which $\dot{\phi}=\omega_{m}$. The left plot also includes two phase comparisons with expansions of different PN order in amplitude, as labeled, for $m \omega_{m}=0.1$. 

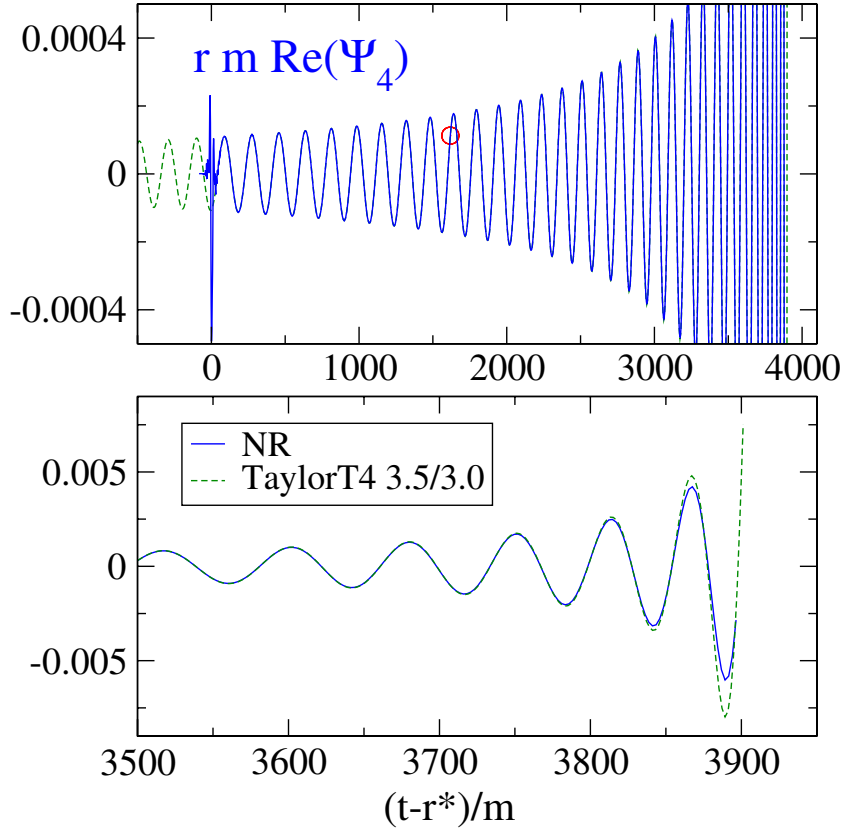

FIG. 20 (color online). Numerical and TaylorT4 3.5/3.0 waveforms. The PN waveform is matched to the numerical one at $m \omega_{m}=0.04$, indicated by the small circle. The lower panel shows a detailed view of the end of the waveform.

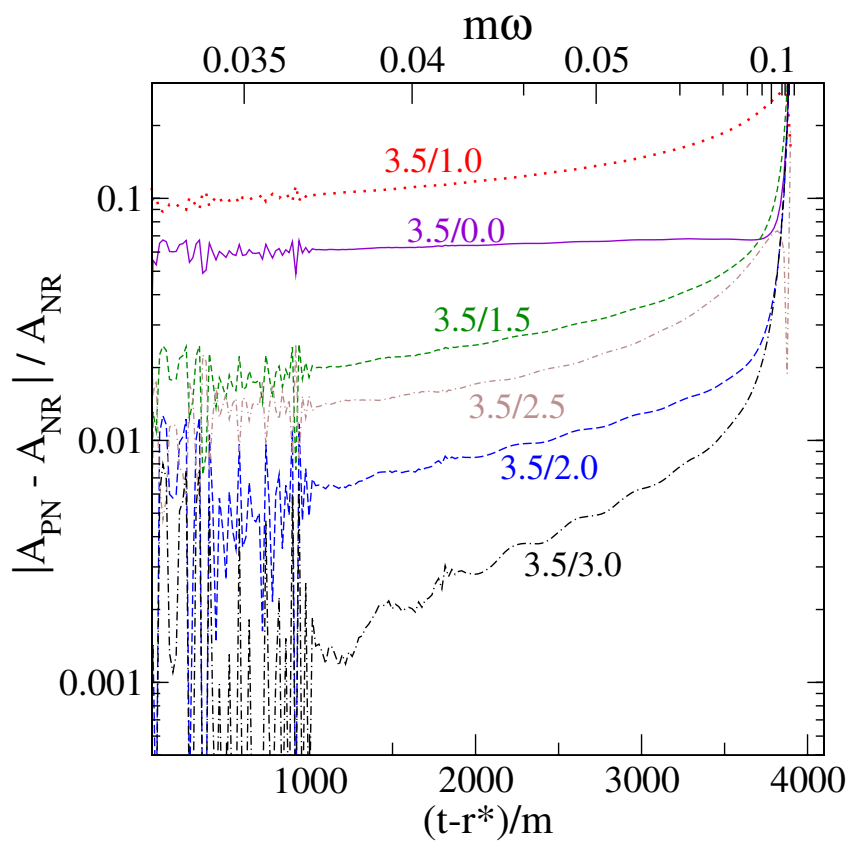

FIG. 21 (color online). TaylorT4 amplitude comparison for different PN orders. Shown is the relative difference in gravitational-wave amplitude between TaylorT4 and numerical $Y_{22}$ waveforms as a function of time. Matching is performed at $m \omega_{m}=0.04$. All curves use 3.5PN order in phase but different PN orders (as labeled) in the amplitude expansion. phase but to 0PN and 3.0PN orders in amplitude, for the case $m \omega_{m}=0.1$. The PN order of the amplitude expansion affects the phase comparison because we are plotting differences in gravitational-wave phase and not orbital phase. The differences between using 0PN, 2.5PN, and 3.0PN amplitude expansions are evident on the scale of the graph, but because these differences are smaller than our estimated uncertainties (see Table III), we cannot reliably conclude which of these most closely agrees with the true waveform.

Figure 21 presents amplitude differences between NR and TaylorT4 as the post-Newtonian order of the amplitude expansion is varied, but the phase expansion remains at 3.5PN. The 2.5PN amplitude curve was already included in the right panel of Fig. 19. We see clearly that higher-order amplitude corrections generally result in smaller differences. The 3PN amplitude correction to the $(2,2)$ mode recently derived by Kidder [62] improves agreement dramatically over the widely known 2.5PN amplitude formulae. Unfortunately, the 3PN amplitude correction to the entire waveform, including all $Y_{l m}$ modes, is not known. ${ }^{7}$

\section{B. Comparing different post-Newtonian approximants}

The previous section presented detailed comparisons of our numerical waveforms with four different postNewtonian approximants. We now turn our attention to some comparisons between these approximants. In this section we also explore further how the post-Newtonian order influences agreement between numerical and postNewtonian waveforms.

Figure 22 presents phase differences as a function of time for all four PN approximants we consider here and for different PN orders. The post-Newtonian and numerical waveforms are matched at $m \omega_{m}=0.04$, about 9 cycles after the beginning of the numerical waveform, and about 21 cycles before its end. We find that some PN approximants at some particular orders agree exceedingly well with the numerical results. The best match is easily TaylorT4 at 3.5PN order, and the next best match is TaylorT4 at 2.0PN order. Some approximants behave significantly worse, such as the TaylorT1 and TaylorT4 waveforms at 2.5PN order. The 2.5PN and 3PN TaylorT3 waveforms agree very well with the numerical waveform at early times, but at late times they accumulate a large phase difference; the $2.5 \mathrm{PN}$ TaylorT3 waveform ends even before the numerical waveform reaches $m \omega=0.1$ (the rightmost vertical brown line in Fig. 22).

We also find that all four PN approximants, when computed to 3PN order or higher, match the numerical wave-

\footnotetext{
${ }^{7}$ To get the complete waveform to $3 \mathrm{PN}$ order, only the $(2,2)$ mode must be known to 3PN order; other modes must be known to smaller PN orders. For an equal-mass, nonspinning binary, all modes except the $(3,2)$ mode are currently known to sufficient order to get a complete 3PN waveform [62].
} 


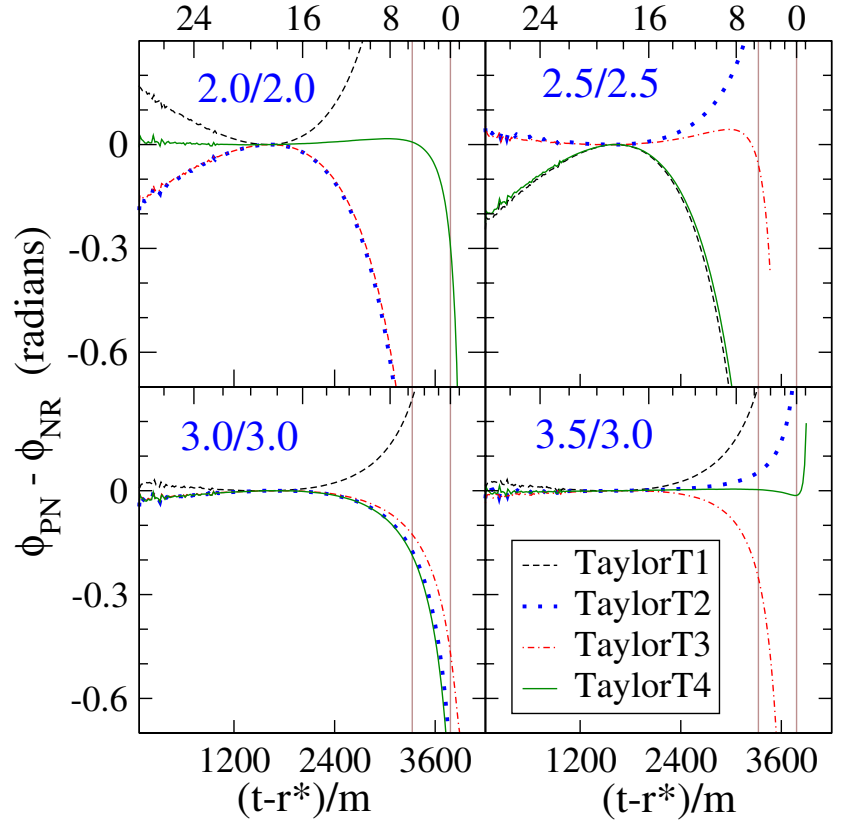

FIG. 22 (color online). Phase comparison for different PN approximants at different PN orders, matched at $m \omega_{m}=0.04$. Shown is the difference in gravitational-wave phase between each post-Newtonian approximant and the numerical $Y_{22}$ waveforms as a function of time. The two vertical brown lines indicate when the numerical simulation reaches $m \omega=0.063$ and 0.1 , respectively; the labels along the top horizontal axis give the number of gravitational-wave cycles before $m \omega=0.1$.

form (and each other) quite closely at early times, when all PN approximants are expected to be accurate. However, at late times, $t-r^{*}>2500 m$, the four PN approximants begin to diverge, indicating that $\mathrm{PN}$ is beginning to break down.

Figure 23 is an enlargement of Fig. 22 for the last 10 gravitational-wave cycles before merger. This figure shows in more detail how the different PN approximants behave near merger.

Figure 24 presents similar results in a different format. We compute the phase differences between the numerical waveform and the various post-Newtonian approximants at the times when the numerical waveform reaches gravitational-wave frequencies $m \omega=0.063$ and $m \omega=$ 0.1 (the times corresponding to these frequencies are also indicated by brown lines in Fig. 22). We then plot these phase differences as a function of the post-Newtonian order (using equal order in phase and amplitude, except for 3.5PN order, where we use 3.0PN in amplitude). Three PN approximants end before $t_{0.1}$ : TaylorT1 2.0/2.0, TaylorT3 2.5/2.5, TaylorT3 3.5/3.0. These data points therefore cannot be included in the right panel of Fig. 24.

The general trend seen in Fig. 24 is that the phase difference decreases with increasing PN order. However, this convergence is not monotonic, and the scatter in Fig. 24 can be larger than the phase differences themselves. For example, the 0PN waveforms are about as good as the

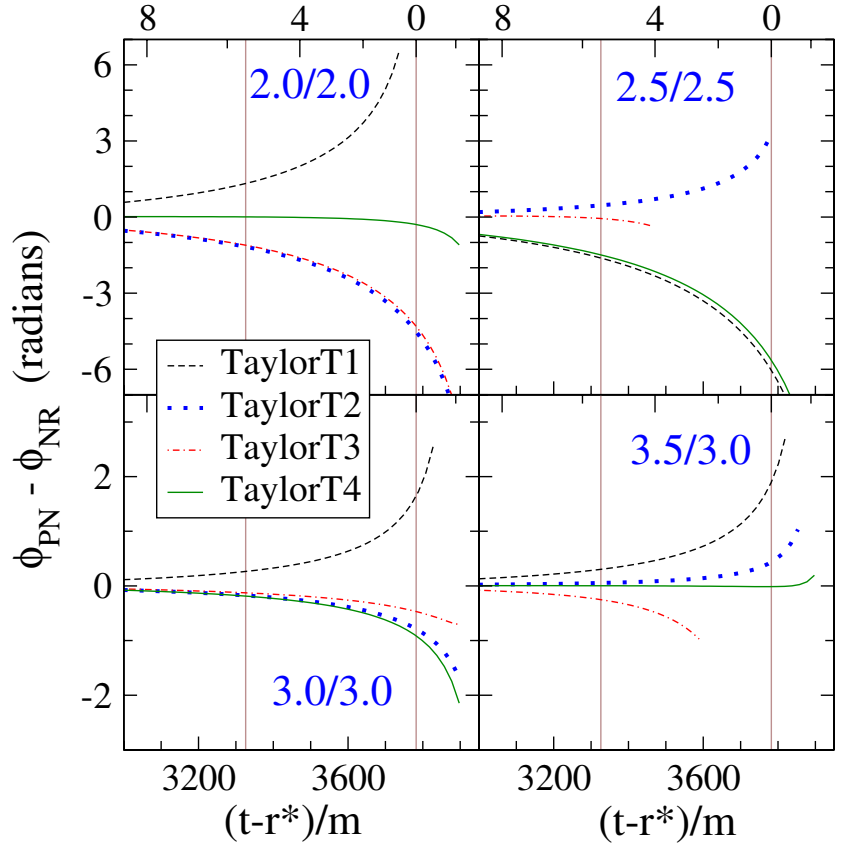

FIG. 23 (color online). Same as Fig. 22, but showing only the last stage of the inspiral. The horizontal axis ends at the estimated time of merger, $\left(t-r^{*}\right)_{\mathrm{CAH}}=3950 m$, cf. Sec II G. The top and bottom panels use different vertical scales.

2.5PN waveforms for TaylorT1 and TaylorT4, and the 2PN TaylorT4 waveform agrees with the numerical results much better than do either the 2.5PN or 3PN TaylorT4

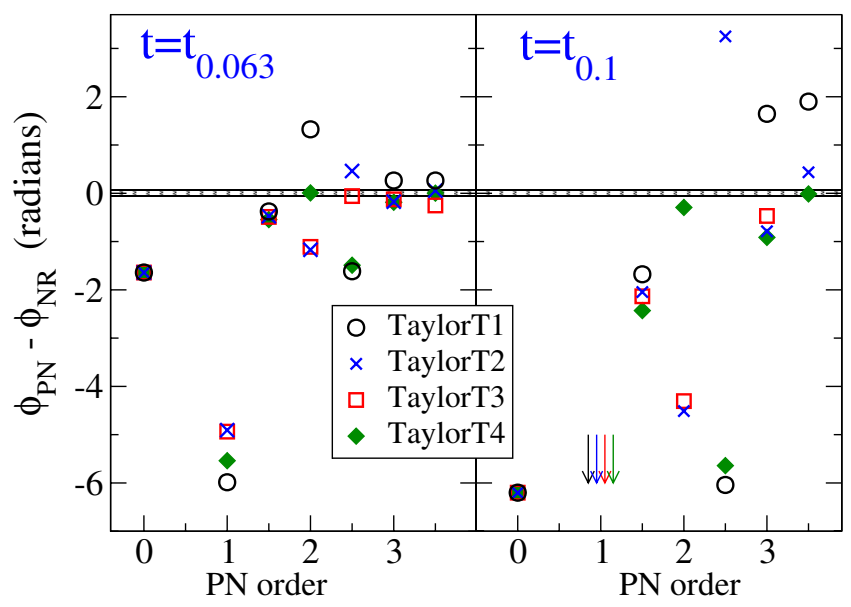

FIG. 24 (color online). Phase differences between numerical and post-Newtonian waveforms at two selected times close to merger. Waveforms are matched at $m \omega_{m}=0.04$, and phase differences are computed at the time when the numerical simulation reaches $m \omega=0.063$ (left panel) and $m \omega=0.1$ (right panel). Differences are plotted versus PN order (equal order in phase and amplitude, except the "3.5 PN" points are 3.5/3.0). On the right plot, the 1PN data points are off scale, clustering at -15 radians. The thin black bands indicate upper bounds on the uncertainty of the comparison as discussed in Sec. VA. 
waveforms. Considering Fig. 24, it seems difficult to make statements about the convergence with PN order for any particular PN approximant, or statements about which PN orders are generally "good." Given that at fixed PN order the different approximants differ merely by the treatment of uncontrolled higher-order terms, the scatter in Fig. 24 in some sense represents the truncation error at each PN order. While some PN approximants at certain orders may show better agreement with the numerical simulation, we are not aware of any means to predict this besides direct comparisons to numerical simulations (as is done here). In particular, Fig. 24 suggests that the remarkable agreement between our numerical results and the 3.5PN TaylorT4 approximant may be simply due to luck; clearly, more PN-NR comparisons are needed, with different mass ratios and spins, to see if this is the case.

\section{CONCLUSIONS}

We have described numerical simulations of an equalmass, nonspinning binary black-hole spacetime covering 15 orbits of inspiral just prior to the merger of the two black holes. Using a multidomain pseudospectral method we are able to extract the gravitational-wave content measured by a distant observer with a phase accuracy of better than 0.02 radians over the roughly 30 cycles of gravitational radiation observed. We demonstrate that in order to achieve this accuracy it is necessary to accurately extrapolate the waveform from data obtained at extraction surfaces sufficiently far from the center of mass of the system. When comparing to zero-spin, zero-eccentricity PN formulae, our phase uncertainty increases to 0.05 radians because the numerical simulation has a small but nonzero orbital eccentricity and small but nonzero spins on the holes.

Judging from the case in which we match at $m \omega_{m}=$ 0.04 , our numerical simulations are consistent (within our estimated phase uncertainty) with all PN approximants (at the highest PN order) from the beginning of our inspiral until about 15 gravitational-wave cycles prior to the merger of the binary. This agreement provides an important validation of our numerical simulation. It also establishes a regime in which the 3.5th order post-Newtonian waveforms are accurate to this level, at least for an equalmass, nonspinning black-hole binary. After this point, the various PN approximants begin to diverge, suggesting that the approximation is beginning to break down. Since there are many different PN approximants (including Padé [54] and effective-one-body $[36,52,58,59]$ which were not discussed in this paper) it may be possible to find a clever way to push the PN expansion beyond its breaking point.

Indeed, we find that one approximant, TaylorT4 at $3.5 \mathrm{PN}$ in phase, works astonishingly well, agreeing with our numerical waveforms for almost the entire 30-cycle length of our runs. Given the wide scatter plot of predictions by various PN approximants, it is likely that TaylorT4 3.5/3.0 simply got lucky for the equal-mass nonspinning black-hole binary. In fact, the assumption of adiabaticity (i.e., circular orbits) is known to lead to much larger phase differences relative to a nonadiabatic inspiral (see Fig. 4 of $[56,143])$ than the phase differences between NR and TaylorT4 we find in Fig. 19. Thus it seems that the uncontrolled higher-order terms of TaylorT4 3.5/3.0 balance the error introduced by the adiabaticity assumption to a remarkable degree. It remains to be determined whether similar cancellations occur when the black-hole masses are unequal or when the holes have nonzero spin.

Regardless of the robustness of TaylorT4, it seems evident that numerical simulations are needed in order to know which, if any, PN approximant yields the correct waveform after the various approximants begin to diverge. For there is no a priori reason why TaylorT4 should be a better choice than TaylorT1 as they differ only in whether the ratio of gravitational-wave flux to the derivative of the orbital energy with respect to frequency is left as a ratio of post-Newtonian expansions or reexpanded as a single postNewtonian expansion.

The surprising accuracy of TaylorT4 3.5/3.0 in the gravitational frequency range from $m \omega=0.035$ through $m \omega=$ 0.15 , for the equal-mass, nonspinning inspiral of two black holes, in principle could form a basis for evaluating the errors of numerical simulations. Instead of worrying about errors due to different formulations, initial data, boundary conditions, extraction methods, etc., perhaps a long inspiral simulation could be compared with TaylorT4 3.5/3.0 in order to get a direct estimate of the phase error. Similarly, because of its good agreement, TaylorT4 3.5/3.0 could also be used to address questions that require much longer waveforms than currently available, for instance the question of when lower order post-Newtonian waveforms become unreliable.

We find that the 3PN contributions to the amplitude of the $(2,2)$ modes improve their accuracy with respect to the numerical waveforms. This suggests that for accurate parameter estimation, it may be desirable to compute the full 3PN amplitude for the polarization waveforms. Despite the formidable nature of the calculation required, it would also be interesting to see how the inclusion of 4PN order corrections to the phasing would affect our comparisons.

Much work still needs to be done to improve the comparison between NR and PN. Our primary goal is to push our simulations through merger and ringdown so that we may compare various resummed PN approximants and the effective-one-body approximants during the last cycle of inspiral and merger, as well as test TaylorT4 3.5/3.0 closer to merger. We also intend to do long inspirals with arbitrary masses and spins in order to test the robustness of PN over a range of these parameters.

Furthermore we wish to improve our initial data. There is a large amount of junk radiation present in the initial data that limits how early we can match PN and NR waveforms. Reduction of this junk radiation [144] would improve the accuracy of our simulations as well. 
Finally, we have done just a simple comparison between NR and PN, without including any treatment of effects that are important for real gravitational-wave detectors such as limited bandwidth and detector noise. In order to more directly address the suitability of PN formulae for analyzing data from gravitational-wave detectors, it will be necessary to fold in the properties of the detector, to consider specific values for the total mass of the binary, and to fit for the mass from the waveforms rather than assuming that the PN and NR waveforms correspond to the same mass. We leave this for future work.

\section{ACKNOWLEDGMENTS}

It is a pleasure to acknowledge useful discussions with Stuart Anderson, Alessandra Buonanno, Mark Hannam, Ian Hinder, Luis Lehner, Lee Lindblom, Geoffrey Lovelace, Sean McWilliams, Robert Owen, Yi Pan, Oliver Rinne, and Kip Thorne. In particular, we would like to thank Alessandra Buonanno for a careful reading of this manuscript, Rob Owen for estimating the BH spin,
Geoffrey Lovelace for his help constructing initial data, Oliver Rinne for providing improved boundary conditions, and Lee Lindblom for his guidance and input throughout this project. This work was supported in part by grants from the Sherman Fairchild Foundation to Caltech and Cornell, and from the Brinson Foundation to Caltech; by NSF Grants No. PHY-0601459, No. PHY-0652995, No. DMS-0553302 and NASA Grant No. NNG05GG52G at Caltech; by NSF Grants No. PHY-0652952, No. DMS0553677, No. PHY-0652929 and NASA Grant No. NNG05GG51G at Cornell; and by the Z. Smith Reynolds Foundation and NSF Grant No. PHY-0555617 at Wake Forest. We thank NASA/JPL for providing computing facilities that contributed to this work. Some of the simulations discussed here were produced with LIGO Laboratory computing facilities. LIGO was constructed by the California Institute of Technology and Massachusetts Institute of Technology with funding from the National Science Foundation and operates under cooperative agreement No. PHY-0107417. This paper has been assigned LIGO document No. LIGO-P070101-00-Z.
[1] F. Pretorius, Phys. Rev. Lett. 95, 121101 (2005).

[2] F. Pretorius, Classical Quantum Gravity 23, S529 (2006).

[3] M. Campanelli, C.O. Lousto, P. Marronetti, and Y. Zlochower, Phys. Rev. Lett. 96, 111101 (2006).

[4] J. G. Baker, J. Centrella, D.-I. Choi, M. Koppitz, and J. van Meter, Phys. Rev. Lett. 96, 111102 (2006).

[5] M. Campanelli, C. O. Lousto, and Y. Zlochower, Phys. Rev. D 73, 061501(R) (2006).

[6] P. Diener, F. Herrmann, D. Pollney, E. Schnetter, E. Seidel, R. Takahashi, J. Thornburg, and J. Ventrella, Phys. Rev. Lett. 96, 121101 (2006).

[7] M. A. Scheel, H. P. Pfeiffer, L. Lindblom, L. E. Kidder, O. Rinne, and S. A. Teukolsky, Phys. Rev. D 74, 104006 (2006).

[8] B. Brügmann, J. A. Gonzalez, M. Hannam, S. Husa, U. Sperhake, and W. Tichy, arXiv:gr-qc/0610128 [Phys. Rev. $\mathrm{D}$ (to be published)].

[9] P. Marronetti, W. Tichy, B. Bruegmann, J. Gonzalez, M. Hannam, S. Husa, and U. Sperhake, Classical Quantum Gravity 24, S43 (2007).

[10] B. Szilágyi, D. Pollney, L. Rezzolla, J. Thornburg, and J. Winicour, Classical Quantum Gravity 24, S275 (2007).

[11] F. Herrmann, I. Hinder, D. Shoemaker, and P. Laguna, Classical Quantum Gravity 24, S33 (2007).

[12] U. Sperhake, Phys. Rev. D 76, 104015 (2007).

[13] Z. B. Etienne, J.A. Faber, Y.T. Liu, S. L. Shapiro, and T. W. Baumgarte, Phys. Rev. D 76, 101503 (2007).

[14] J. G. Baker, J. Centrella, D.-I. Choi, M. Koppitz, and J. van Meter, Phys. Rev. D 73, 104002 (2006).

[15] J.G. Baker, M. Campanelli, F. Pretorius, and Y. Zlochower, Classical Quantum Gravity 24, S25 (2007).

[16] J. G. Baker, J. Centrella, D.-I. Choi, M. Koppitz, J. R. van
Meter, and M. C. Miller, Astrophys. J. 653, L93 (2006).

[17] J. A. Gonzalez, U. Sperhake, B. Brügmann, M. Hannam, and S. Husa, Phys. Rev. Lett. 98, 091101 (2007).

[18] M. Koppitz, D. Pollney, C. Reisswig, L. Rezzolla, J. Thornburg, P. Diener, and E. Schnetter, Phys. Rev. Lett. 99, 041102 (2007).

[19] M. Campanelli, C. O. Lousto, Y. Zlochower, and D. Merritt, Phys. Rev. Lett. 98, 231102 (2007).

[20] J.A. Gonzalez, M.D. Hannam, U. Sperhake, B. Brügmann, and S. Husa, Phys. Rev. Lett. 98, 231101 (2007).

[21] F. Herrmann, I. Hinder, D. Shoemaker, P. Laguna, and R. A. Matzner, Astrophys. J. 661, 430 (2007).

[22] C. F. Sopuerta, N. Yunes, and P. Laguna, Astrophys. J. 656, L9 (2007).

[23] D.-I. Choi, B. J. Kelly, W.D. Boggs, J. G. Baker, J. Centrella, and J. van Meter, Phys. Rev. D 76,104026 (2007).

[24] M. Campanelli, C. O. Lousto, Y. Zlochower, and D. Merritt, Astrophys. J. 659, L5 (2007).

[25] B. Brügmann, J. Gonzalez, M. Hannam, S. Husa, and U. Sperhake, arXiv:0707.0135.

[26] J. G. Baker, W. D. Boggs, J. Centrella, B. J. Kelly, S. T. McWilliams, M. C. Miller, and J. R. van Meter, Astrophys. J. 668, 1140 (2007).

[27] F. Herrmann, I. Hinder, D. M. Shoemaker, P. Laguna, and R. A. Matzner, Phys. Rev. D 76, 084032 (2007).

[28] J.D. Schnittman, A. Buonanno, J. R. van Meter, J. G. Baker, W. D. Boggs, J. Centrella, B.J. Kelly, and S. T. McWilliams, arXiv:0707.0301v1.

[29] M. Campanelli, C. O. Lousto, Y. Zlochower, and D. Merritt, Phys. Rev. D 75, 064030 (2007). 
[30] M. Campanelli, C. O. Lousto, and Y. Zlochower, Phys. Rev. D 74, 084023 (2006).

[31] M. Campanelli, C. O. Lousto, and Y. Zlochower, Phys. Rev. D 74, 041501 (2006).

[32] A. Buonanno, G. B. Cook, and F. Pretorius, Phys. Rev. D 75, 124018 (2007).

[33] P. Ajith, S. Babak, Y. Chen, M. Hewitson, B. Krishnan, J. T. Whelan, B. Bruegmann, P. Diener, J. Gonzalez, and M. Hannam et al., Classical Quantum Gravity 24, S689 (2007).

[34] E. Berti, V. Cardoso, J.A. Gonzalez, U. Sperhake, M. Hannam, S. Husa, and B. Bruegmann, Phys. Rev. D 76, 064034 (2007).

[35] Y. Pan, A. Buonanno, J. G. Baker, J. Centrella, B. J. Kelly, S. T. McWilliams, F. Pretorius, and J.R. van Meter, arXiv:0704.1964v1 [Phys. Rev. D (to be published)].

[36] A. Buonanno, Y. Pan, J. G. Baker, J. Centrella, B. J. Kelly, S. T. McWilliams, and J. R. van Meter, Phys. Rev. D 76, 104049 (2007).

[37] T. Baumgarte, P. Brady, J.D. Creighton, L. Lehner, F. Pretorius, and R. DeVoe, arXiv:gr-qc/0612100.

[38] J. G. Baker, J.R. van Meter, S.T. McWilliams, J. Centrella, and B.J. Kelly, Phys. Rev. Lett. 99, 181101 (2007).

[39] J. G. Baker, S.T. McWilliams, J.R. van Meter, J. Centrella, D.-I. Choi, B. J. Kelly, and M. Koppitz, Phys. Rev. D 75, 124024 (2007).

[40] H. P. Pfeiffer, D. A. Brown, L. E. Kidder, L. Lindblom, G. Lovelace, and M. A. Scheel, Classical Quantum Gravity 24, S59 (2007).

[41] M. Hannam, S. Husa, U. Sperhake, B. Brügmann, and J. A. Gonzalez, arXiv:0706.1305v2.

[42] S. Husa, J. A. Gonz'alez, M. Hannam, B. Brügmann, and U. Sperhake, arXiv:0706.0740v1.

[43] B. C. Barish and R. Weiss, Phys. Today 52, 44 (1999).

[44] S. J. Waldman, Classical Quantum Gravity 23, S653 (2006).

[45] S. Hild, Classical Quantum Gravity 23, S643 (2006).

[46] F. Acernese et al., Classical Quantum Gravity 19, 1421 (2002).

[47] F. Acernese, P. Amico, M. Alshourbagy, F. Antonucci, S. Aoudia, S. Avino, D. Babusci, G. Ballardin, F. Barone, and L. Barsotti et al., Classical Quantum Gravity 23, S635 (2006).

[48] P. Fritschel, Proc. SPIE-Int. Soc. Opt. Eng. 4856, 282 (2003).

[49] E. E. Flanagan and S. A. Hughes, Phys. Rev. D 57, 4535 (1998).

[50] P. C. Peters, Phys. Rev. 136, B1224 (1964).

[51] T. Damour, B. R. Iyer, and B. S. Sathyaprakash, Phys. Rev. D 57, 885 (1998).

[52] A. Buonanno and T. Damour, Phys. Rev. D 59, 084006 (1999).

[53] A. Buonanno, Y.-B. Chen, and M. Vallisneri, Phys. Rev. D 67, 024016 (2003); 74, 029903(E) (2006).

[54] T. Damour, B. R. Iyer, and B. S. Sathyaprakash, Phys. Rev. D 63, 044023 (2001); 72, 029902(E) (2005).

[55] T. Damour, B. R. Iyer, and B. S. Sathyaprakash, Phys. Rev. D 66, 027502 (2002); 72, 029901(E) (2005).

[56] A. Buonanno and T. Damour, Phys. Rev. D 62, 064015 (2000).
[57] T. Damour, P. Jaranowski, and G. Schäfer, Phys. Rev. D 62, 084011 (2000).

[58] T. Damour, Phys. Rev. D 64, 124013 (2001).

[59] T. Damour, B. R. Iyer, P. Jaranowski, and B.S. Sathyaprakash, Phys. Rev. D 67, 064028 (2003).

[60] A. Buonanno, Y. Chen, and T. Damour, Phys. Rev. D 74, 104005 (2006).

[61] S. Husa, M. Hannam, J. A. González, U. Sperhake, and B. Brügmann, arXiv:0706.0904.

[62] L. E. Kidder, arXiv:0710.0614.

[63] J. W. York, Jr., Phys. Rev. Lett. 82, 1350 (1999).

[64] H. P. Pfeiffer and J. W. York, Phys. Rev. D 67, 044022 (2003).

[65] H. P. Pfeiffer, L.E. Kidder, M. A. Scheel, and S. A. Teukolsky, Comput. Phys. Commun. 152, 253 (2003).

[66] G. B. Cook, Phys. Rev. D 65, 084003 (2002).

[67] G. B. Cook and H.P. Pfeiffer, Phys. Rev. D 70, 104016 (2004).

[68] M. Caudill, G. B. Cook, J. D. Grigsby, and H. P. Pfeiffer, Phys. Rev. D 74, 064011 (2006).

[69] L. Lindblom, M. A. Scheel, L. E. Kidder, R. Owen, and O. Rinne, Classical Quantum Gravity 23, S447 (2006).

[70] H. Friedrich, Commun. Math. Phys. 100, 525 (1985).

[71] D. Garfinkle, Phys. Rev. D 65, 044029 (2002).

[72] F. Pretorius, Classical Quantum Gravity 22, 425 (2005).

[73] O. Rinne, Classical Quantum Gravity 23, 6275 (2006).

[74] O. Rinne, L. Lindblom, and M. A. Scheel, Classical Quantum Gravity 24, 4053 (2007).

[75] J. M. Stewart, Classical Quantum Gravity 15, 2865 (1998).

[76] H. Friedrich and G. Nagy, Commun. Math. Phys. 201, 619 (1999).

[77] J. M. Bardeen and L. T. Buchman, Phys. Rev. D 65, 064037 (2002).

[78] B. Szilágyi, B. Schmidt, and J. Winicour, Phys. Rev. D 65, 064015 (2002).

[79] G. Calabrese, J. Pullin, O. Sarbach, M. Tiglio, and O. Reula, Commun. Math. Phys. 240, 377 (2003).

[80] B. Szilágyi and J. Winicour, Phys. Rev. D 68, 041501(R) (2003).

[81] L. E. Kidder, L. Lindblom, M. A. Scheel, L. T. Buchman, and H. P. Pfeiffer, Phys. Rev. D 71, 064020 (2005).

[82] L. T. Buchman and O.C. A. Sarbach, Classical Quantum Gravity 23, 6709 (2006).

[83] M. Bjørhus, SIAM J. Sci. Comput. 16, 542 (1995).

[84] D. Gottlieb and J. S. Hesthaven, J. Comput. Appl. Math. 128, 83 (2001).

[85] J.S. Hesthaven, Applied Numerical Mathematics 33, 23 (2000).

[86] C. Gundlach, Phys. Rev. D 57, 863 (1998).

[87] T. W. Baumgarte, G. B. Cook, M. A. Scheel, S. L. Shapiro, and S. A. Teukolsky, Phys. Rev. D 54, 4849 (1996).

[88] H. P. Pfeiffer, S. A. Teukolsky, and G. B. Cook, Phys. Rev. D 62, 104018 (2000).

[89] J. D. Brown and J. W. York, Phys. Rev. D 47, 1407 (1993).

[90] A. Ashtekar, C. Beetle, and J. Lewandowsky, Phys. Rev. D 64, 044016 (2001).

[91] A. Ashtekar and B. Krishnan, Phys. Rev. D 68, 104030 (2003).

[92] O. Dreyer, B. Krishnan, E. Schnetter, and D. Shoemaker, Phys. Rev. D 67, 024018 (2003).

[93] G. B. Cook and B. F. Whiting, Phys. Rev. D 76, 041501(R) 
(2007).

[94] R. Owen, Ph.D. thesis, California Institute of Technology, 2007; http://resolver.caltech.edu/CaltechETD:etd05252007-143511.

[95] D. R. Fiske, J. G. Baker, J. R. van Meter, D.-I. Choi, and J. M. Centrella, Phys. Rev. D 71, 104036 (2005).

[96] C. Beetle, M. Bruni, L. M. Burko, and A. Nerozzi, Phys. Rev. D 72, 024013 (2005).

[97] A. Nerozzi, C. Beetle, M. Bruni, L. M. Burko, and D. Pollney, Phys. Rev. D 72, 024014 (2005).

[98] L. M. Burko, T. W. Baumgarte, and C. Beetle, Phys. Rev. D 73, 024002 (2006).

[99] M. Campanelli, B. J. Kelly, and C. O. Lousto, Phys. Rev. D 73, 064005 (2006).

[100] A. Nerozzi, M. Bruni, L. M. Burko, and V. Re, AIP Conf. Proc. 861, 702 (2006).

[101] E. Pazos, E. N. Dorband, A. Nagar, C. Palenzuela, E. Schnetter, and M. Tiglio, Classical Quantum Gravity 24, S341 (2007).

[102] L. Lehner and O. M. Moreschi, arXiv:0706.1319 [Phys. Rev. D (to be published) \}.

[103] B. Kocsis and A. Loeb, Phys. Rev. D 76, 084022 (2007).

[104] L. Blanchet, Living Rev. Relativity 9, 4 (2006).

[105] P. Jaranowski and G. Schäfer, Phys. Rev. D 57, 7274 (1998); 63, 029902(E) (2000).

[106] P. Jaranowski and G. Schäfer, Phys. Rev. D 60, 124003 (1999).

[107] T. Damour, P. Jaranowski, and G. Schäfer, Phys. Rev. D 62, 021501(R) (2000); 63, 029903(E) (2000).

[108] T. Damour, P. Jaranowski, and G. Schäfer, Phys. Rev. D 63, 044021 (2001); 66, 029901(E) (2002).

[109] L. Blanchet and G. Faye, Phys. Lett. A 271, 58 (2000).

[110] L. Blanchet and G. Faye, Phys. Rev. D 63, 062005 (2001).

[111] T. Damour, P. Jaranowski, and G. Schäfer, Phys. Lett. B 513, 147 (2001).

[112] L. Blanchet, T. Damour, and G. Esposito-Farèse, Phys. Rev. D 69, 124007 (2004).

[113] Y. Itoh, T. Futamase, and H. Asada, Phys. Rev. D 63, 064038 (2001).

[114] Y. Itoh and T. Futamase, Phys. Rev. D 68, 121501(R) (2003).

[115] Y. Itoh, Phys. Rev. D 69, 064018 (2004).

[116] L. Blanchet and B. R. Iyer, Classical Quantum Gravity 20, 755 (2003).

[117] V. C. de Andrade, L. Blanchet, and G. Faye, Classical Quantum Gravity 18, 753 (2001).

[118] K. S. Thorne, Rev. Mod. Phys. 52, 299 (1980).
[119] L. Blanchet, Classical Quantum Gravity 15, 1971 (1998).

[120] L. Blanchet and T. Damour, Phys. Rev. D 46, 4304 (1992).

[121] L. Blanchet, Classical Quantum Gravity 15, 89 (1998).

[122] L. Blanchet, Classical Quantum Gravity 15, 113 (1998); 22, 3381(E) (2005).

[123] C. Cutler, T. A. Apostolatos, L. Bildsten, L. S. Finn, E. E. Flanagan, D. Kennefick, D. M. Markovic, A. Ori, E. Poisson, and G. J. Sussman et al., Phys. Rev. Lett. 70, 2984 (1993).

[124] L. Blanchet, B. R. Iyer, and B. Joguet, Phys. Rev. D 65, 064005 (2002); 71, 129903(E) (2005).

[125] L. Blanchet, G. Faye, B. R. Iyer, and B. Joguet, Phys. Rev. D 65, 061501(R) (2002); 71, 129902(E) (2005).

[126] L. Blanchet and B. R. Iyer, Phys. Rev. D 71, 024004 (2005).

[127] L. Blanchet, T. Damour, G. Esposito-Farèse, and B. R. Iyer, Phys. Rev. Lett. 93, 091101 (2004).

[128] L. Blanchet, T. Damour, G. Esposito-Farèse, and B. R. Iyer, Phys. Rev. D 71, 124004 (2005).

[129] K. Arun, L. Blanchet, B. Iyer, and M. Qusailah, Classical Quantum Gravity 21, 3771 (2004); 22, 3115(E) (2005).

[130] L.E. Kidder, L. Blanchet, and B. R. Iyer, Classical Quantum Gravity 24, 5307 (2007).

[131] A. G. Wiseman, Phys. Rev. D 48, 4757 (1993).

[132] L. Blanchet and G. Schäfer, Classical Quantum Gravity 10, 2699 (1993).

[133] L. Blanchet, Phys. Rev. D 54, 1417 (1996).

[134] L. Blanchet, Phys. Rev. D 55, 714 (1997).

[135] E. Poisson, Phys. Rev. D 70, 084044 (2004).

[136] A. Królak, K. D. Kokkotas, and G. Schäfer, Phys. Rev. D 52, 2089 (1995).

[137] H. Wahlquist, Gen. Relativ. Gravit. 19, 1101 (1987).

[138] G. Faye, L. Blanchet, and A. Buonanno, Phys. Rev. D 74, 104033 (2006).

[139] L. Blanchet, A. Buonanno, and G. Faye, Phys. Rev. D 74, 104034 (2006); 75, 049903(E) (2007).

[140] L. E. Kidder, Phys. Rev. D 52, 821 (1995).

[141] C. M. Will and A. G. Wiseman, Phys. Rev. D 54, 4813 (1996).

[142] LSC Algorithm Library software packages LAL, LALWRAPPER, and LALAPPS, http://www.lsc-group. phys.uwm.edu/lal.

[143] M. Miller, Phys. Rev. D 69, 124013 (2004).

[144] G. M. Lovelace, Ph.D. thesis, California Institute of Technology, 2007; http://etd.caltech.edu/etd/available/ etd-05232007-115433. 